\title{
Units of plasticity in bacterial genomes: new insight from the comparative genomics of two bacteria interacting with invertebrates, Photorhabdus and Xenorhabdus
}

\author{
Jean-Claude Ogier ${ }^{1,2}$, Alexandra Calteau ${ }^{3}$, Steve Forst ${ }^{4}$, Heidi Goodrich-Blair ${ }^{5}$, David Roche ${ }^{3}$, Zoé Rouy ${ }^{3}$, \\ Garret Suen ${ }^{6}$, Robert Zumbihl ${ }^{1,2}$, Alain Givaudan ${ }^{1,2}$, Patrick Tailliez $^{1,2}$, Claudine Médigue ${ }^{3}$, Sophie Gaudriault ${ }^{1,2^{*}}$
}

\begin{abstract}
Background: Flexible genomes facilitate bacterial evolution and are classically organized into polymorphic strainspecific segments called regions of genomic plasticity (RGPs). Using a new web tool, RGPFinder, we investigated plasticity units in bacterial genomes, by exhaustive description of the RGPs in two Photorhabdus and two Xenorhabdus strains, belonging to the Enterobacteriaceae and interacting with invertebrates (insects and nematodes).

Results: RGPs account for about $60 \%$ of the genome in each of the four genomes studied. We classified RGPs into genomic islands (Gls), prophages and two new classes of RGP without the features of classical mobile genetic elements (MGEs) but harboring genes encoding enzymes catalyzing DNA recombination (RGP mob), or with no remarkable feature ( $R_{\text {none }}$ ). These new classes accounted for most of the RGPs and are probably hypervariable regions, ancient MGEs with degraded mobilization machinery or non canonical MGEs for which the mobility mechanism has yet to be described. We provide evidence that not only the Gls and the prophages, but also

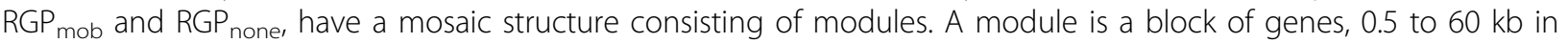
length, displaying a conserved genomic organization among the different Enterobacteriaceae. Modules are functional units involved in host/environment interactions (22-31\%), metabolism (22-27\%), intracellular or intercellular DNA mobility (13-30\%), drug resistance (4-5\%) and antibiotic synthesis (3-6\%). Finally, in silico comparisons and PCR multiplex analysis indicated that these modules served as plasticity units within the bacterial genome during genome speciation and as deletion units in clonal variants of Photorhabdus.
\end{abstract}

Conclusions: This led us to consider the modules, rather than the entire RGP, as the true unit of plasticity in bacterial genomes, during both short-term and long-term genome evolution.

\section{Background}

The portion of the bacterial genome common to all strains in a defined set of species and required for basic cellular functions is known as the core genome. The genes variably present between individual strains constitute the flexible genome [1-3]. The estimate of the core and the flexible genomes not only depend on the phylogenetic depth of the group considered, the number of

\footnotetext{
* Correspondence: sgaudriault@univ-montp2.fr

'INRA, UMR 1133, Laboratoire EMIP, Place Eugène Bataillon, F-34095

Montpellier, France

Full list of author information is available at the end of the article
}

genomes available for comparison but also on the methodology used [3]. Some genes of the flexible genome may play a role in adaptation to special growth conditions, such as those involved in the colonization of new ecological niches, symbiosis, host-cell interaction, and pathogenicity $[1,2]$. The plasticity of the flexible genome contributes to bacterial genome evolution [2-4].

The flexible genome is organized principally into polymorphic strain-specific DNA segments that are missing in at least one of the genomes analyzed. These segments are named regions of genomic plasticity (RGPs) without any assumption about the evolutionary origin or genetic 
basis of these variable chromosomal segments [5]. This terminology covers two classes: hypervariable segments that are likely to be the result of deletions of particular DNA regions in one or more strains, and the mobile genetic elements (MGEs).

The plasticity of MGEs depends on three kinds of molecular events, the duplications, inversions and deletions, mediated by transposases and site-specific recombinases whose genes are located either on core genome or on the RGPs themselves [4,6]. The MGEs may be excised from one location and reintegrated elsewhere in the genome or may undergo replicative transposition before integration of a new copy of the element elsewhere in the genome (intracellular mobility). Finally, some MGEs may undergo horizontal genetic transfer (HGT) by natural transformation, transduction or well developed and efficient conjugation mechanisms (intercellular mobility) [4].

The MGE class covers some well characterized elements. Plasmids are stable self-replicating MGEs [4]. Some of them may be transferred in other prokaryotic cells by conjugation. Prophages, the integrated form of temperate bacteriophages, are MGEs that undergo intercellular DNA mobility via transduction [4]. Non replicative MGEs are integrated into the host chromosome and encode at least one enzyme involved in their own excision and integration; these MGEs constitute a large, diverse family $[7,8]$. They are referred to as (i) transposable elements, if they do not undergo HGT, (ii) genomic islands (GI) if they present features of HGT (phage and/or plasmid-derived sequences, transfer genes, integrases, insertion sequences (IS), $\mathrm{G}+\mathrm{C}$ content and codon usage bias) but do not encode genes involved in transfer, (iii) integrative mobilizable elements when they require "helper" elements for mobilization and (iv) integrative conjugative elements (ICEs) when they encode their own complete mobility machinery, generally a type 4 secretion system (T4SS) [8]. However, it is often difficult to apply this nomenclature, because MGEs are generally described on the basis of in silico analysis in large-scale prokaryotic genome sequencing programs. Thus, the effective excision, intracellular or intercellular mobility and subsequent reintegration via site-specific recombination of MGEs have been demonstrated in only a few cases [9-14]. For these reasons, in the course of genomic projects, in the absence of experimental data, the term "GI" is generally used for putative mobilizable MGEs without the organization typical of prophages $[1,2,4,15]$.

MGEs are potent agents of bacterial genome evolution $[4,16]$. This property results from both the plasticity of MGE and intra-MGE recombination. Indeed, some MGEs are organized into an array of MGE sub-segments, known as modules $[7,17,18]$. This mosaic organization is the product of the combination of a limited number of constitutive modules [17]: intracellular mobility modules (recombination and replication functions), intercellular mobility modules (transformation, phage propagation and conjugative transfer) and stability modules. The stability modules are responsible for the maintenance of the MGE in the host cell and encode functions such as poison/antidote systems [19] and antibiotic resistance functions [20,21]. Recombination between MGEs has been studied in a few cases. Deletions and tandem accretions of modules generate hybrid MGEs [22-24]. The bacterial recA gene or the recombination systems of the MGEs themselves may mediate the generation of hybrid MGEs [25].

We investigated the plasticity of the flexible genome, by addressing three questions: what are the respective roles of MGEs and hypervariable segments within the flexible genome? Are all RGPs, like MGEs, composed of modules? Do all modules undergo accretion? We addressed these questions by studying the flexible genomes of Photorhabdus and Xenorhabdus. Photorhabdus and Xenorhabdus are closely related Enterobacteriaceae [26], both of which are appropriate for genomic evolution studies because of their particular lifestyle $[27,28]$. Photorhabdus and Xenorhabdus live in monoxenic cultures within the gut of the soil nematodes, Heterorhabditis and Steinernema, respectively. These nematodes infect insect larvae, releasing the bacteria into the hemolymph of the insect. The nematode and the bacteria kill the insect and convert the cadaver into a source of food for nematode growth and development. After several rounds of reproduction, the bacteria recolonize the nematodes, which then emerge from the insect cadaver into the soil, to search for a new host [29-31]. This lifestyle, including obligatory, cyclic pathogenic and mutualistic interactions with invertebrate hosts, restricts the ecological niches colonized by Photorhabus and Xenorhabdus. This biological constraint may favor clonality among bacteria and intrachromosomal rearrangements within the genome. Moreover, Photorhabdus asymbiotica has been recovered in clinical isolates from human wounds, in both North America and Australia [32,33]. The emergence of pathogenicity in humans is also consistent with a potential for genomic exchange with environmental bacteria.

Genomic plasticity has been studied to different extents in the two genera. Whole-genome analysis has just begun for Xenorhabdus [34], whereas full genome sequences have been published for two Photorhabdus strains, revealing the presence of a large number of phage remnants, IS, transposases, repeat elements and overrepresented families of paralogs, consistent with a high level of potential plasticity in these genomes $[35,36]$. These features are indicative of a general 
process of genome evolution, as repeatedly observed in host-restricted lineages from many phylogenetic groups [37]. One study described genomic deletion and amplification events in Photorhabdus clonal variants obtained in laboratory conditions [38]. These genomic changes are cryptic, but are always found within the Enterobacteriaceae flexible genome. Finally, some studies have characterized a few Photorhabdus and Xenorhabdus RGPs by in silico analysis [35,36,39-43] or by microarray hybridization $[38,44]$.

In this study, we carried out an exhaustive description of RGPs in the genomes of three entomopathogenic strains isolated from nematodes: Xenorhabdus nematophila ATCC19061 [34], Xenorhabdus bovienii SS-2004 [34] and Photorhabdus luminescens TT01 [35], and a strain isolated from humans: Photorhabdus asymbiotica ATCC43949 [36]. For the identification of hypervariable regions, recent MGEs, ancient MGEs and non canonical MGEs with unknown mobility mechanisms, we used a new Web tool, RGPFinder, which identifies both synteny ruptures in the core genome and classical intrinsic and extrinsic MGE features (Roche, D., unpublished data). We then described the fine modular structure of RGPs and showed that (i) each module is a functional unit, (ii) modules have diverse functions, (iii) modules shape the flexible genomes of the various strains studied and, (iv) some modules are deletion units. Overall, our data strongly suggest that modules are the functional integrated systems serving as the real unit of plasticity within RGPs.

\section{Results and Discussion}

\section{Identification of regions of genomic plasticity (RGPs)}

The size of the flexible genome depends on the methodology used, the depth of phylogenetic comparison and the number of genomes compared [3]. Methods based on genomic comparison, detection of composition bias and search of mobility genes are the most performing tools for the flexible genome characterization [45]. Some methods such as IslandViewer and MobileHomeFinder are dedicated to predict genomic islands (GIs) with high stringency. Our goal is the identification of the regions of genomic plasticity (RGPs), which covers not only GIs but also rearrangement events without any assumption about the evolutionary origin or genetic basis of these variable chromosomal segments. For this reason, we developed a new Web tool, RGPFinder, which combines comparison and composition based approaches (Roche et al., unpublished data). Furthermore, RGPFinder is specifically designed to identify regions absent from at least one genome inside the comparison genome set. We applied this tool on the genomes of Photorhabdus luminescens TT01 (Pl), Photorhabdus asymbiotica ATCC43949 (Pa), Xenorhabdus nematophila ATCC19061 (Xn) and Xenorhabdus bovienii SS-2004 (Xb) strains. We compared the results of IslandViewer and RGPFinder on our four genomes (data not shown). RGPFinder carries out a larger description of the flexible genome (more and larger predicted regions) than IslandViewer.

We applied the RGPFinder method to different bacterial genome sets (Figure 1.A and Table 1). The first set included strains from the same genus ("Genus" set: Xn versus $\mathrm{Xb}$ and $\mathrm{Pl}$ versus $\mathrm{Pa}$ ). The second set included the four Photorhabdus and Xenorhabdus strains ("Photo + Xeno" set). The third set, the "Entero" set, included Photorhabdus and Xenorhabus strains together with other closely related strains from the Enterobacteriaceae (Additional file 1), including the mammalian pathogen Salmonella enterica subsp. enterica Typhi CT18, the mammalian pathogen Yersinia pestis CO92, which also interacts with insects, the plant pathogen, Erwinia carotovora subsp. atroseptica SCRI1043, and a commensal strain, Escherichia coli K12. The proportion of the genome accounted for by RGPs increased with the size of the bacterial genome set: $26-36 \%, 46-58 \%$ and $60-69 \%$ for the Genus, Photo + Xeno and Entero sets (Table 1). However, the number of RGPs was similar in the different bacterial genome sets. The nucleus of each RGP was conserved, but was larger when the "Entero" set was used. The characterization of flexible genomes through the comparison of closely related strains can be used to identify RGPs that have recently been acquired or modified, but this technique may result in RGPs generated by more ancient HGT or rearrangements being incorrectly considered part of the core genome[5]. As our objective was the exhaustive characterization of flexible genome plasticity and identification of as many HGT and rearrangement events as possible, we chose to work on the largest comparison set, the Entero set.

After manual inspection of the predicted RGPs (see Methods), we obtained a list of 96, 92, 83 and 71 RGPs sensu lato for $\mathrm{Pl}, \mathrm{Pa}, \mathrm{Xn}$ and $\mathrm{Xb}$, respectively (Table 1 and see RGPs listed in Additional File 2; these lists are the references used throughout this work.). The RGPs were between $5 \mathrm{~kb}$ and $316 \mathrm{~kb}$ in length, and more than $50 \%$ were less than $20 \mathrm{~kb}$ long (Additional File 3). No integral RGP was found to be conserved in all four genomes. The flexible genome of the Photorhabdus and Xenorhabdus genera accounted for 52.6 to $61.5 \%$ of the entire genome (Table 1). In other studies in conditions similar to those used here, the flexible genome has been found to cover: i) 1 to $10 \%$ of the genome when serovar or clinical isolates are compared [5,46]; ii) 10 to $40 \%$ of the genome when strains are compared $[47,48]$, iii) 25 to $60 \%$ of the genome when species are compared [49-51] and iv) 50 to $69 \%$ of the genome when genera are compared [52]. Thus, the sizes of the flexible 


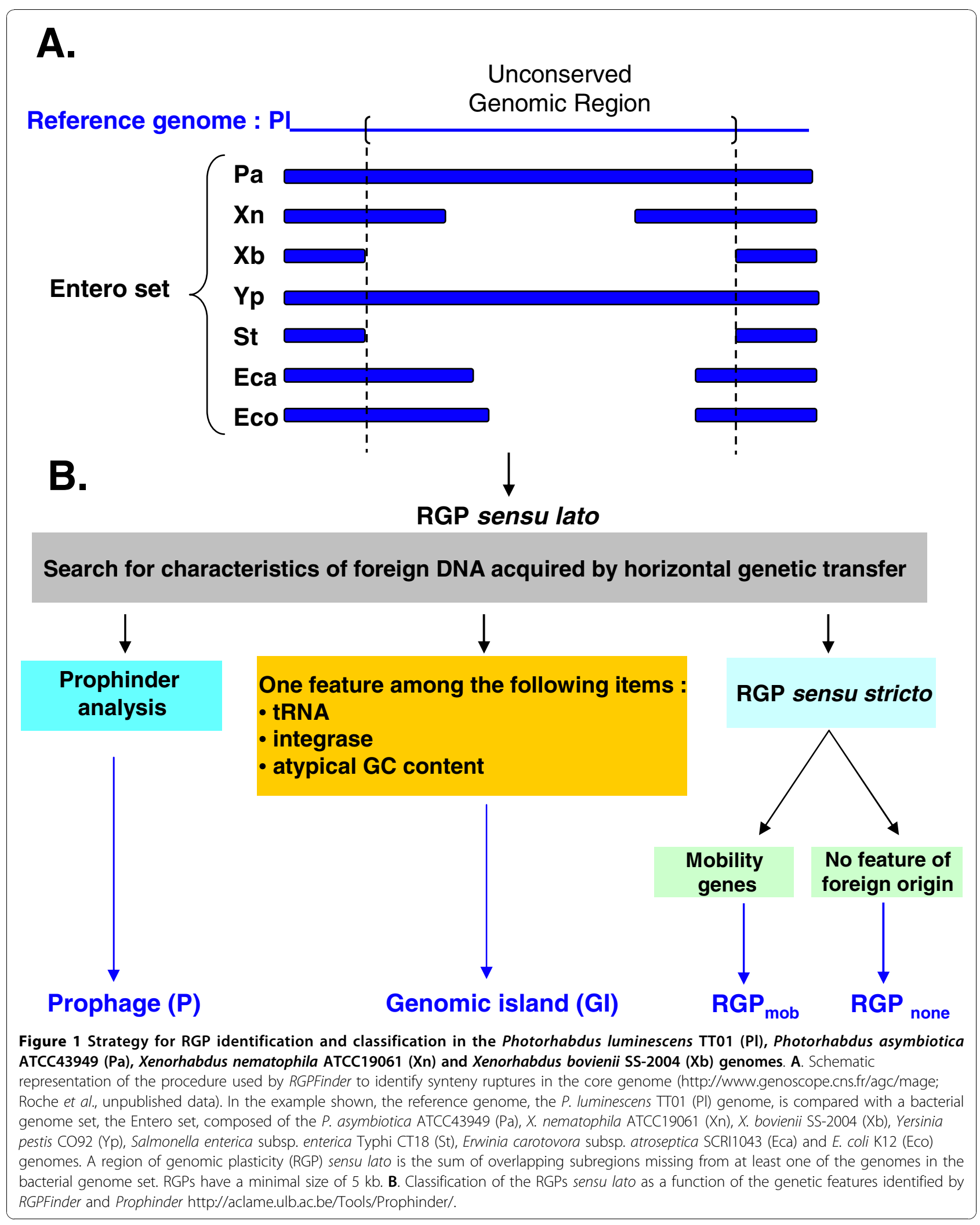


Table 1 Number and size of regions of genomic plasticity (RGPs) in the $P$. luminescens TT01, $P$. asymbiotica ATCC43949, $X$. nematophila ATCC19061 and X. bovienii SS-2004 genomes, according to the set of bacterial genomes used to search for synteny ruptures in the core genome

\begin{tabular}{|c|c|c|c|c|c|}
\hline & \multirow{2}{*}{$\begin{array}{l}\text { Genome } \\
\text { size (bp) }\end{array}$} & \multicolumn{4}{|c|}{ Number of predicted RGPs (\% of the whole genome), when compared with the } \\
\hline & & "Genus" set ${ }^{1 *}$ & "Photo + Xeno" set ${ }^{2 *}$ & "Entero" set ${ }^{3 *}$ & "Entero" set after cleaning** \\
\hline P. luminescens TT01 & 5688987 & $111(32.8 \%)$ & $122(58.2 \%)$ & $113(69 \%)$ & $96(61.5 \%)$ \\
\hline X. nematophila ATCC19061 & 4432590 & $96(36.2 \%)$ & $95(49.5 \%)$ & $97(62.9 \%)$ & $83(54.1 \%)$ \\
\hline X. bovienii SS-2004 & 4225498 & $81(33.1 \%)$ & $80(45.8 \%)$ & $88(59.6 \%)$ & $71(52.6 \%)$ \\
\hline
\end{tabular}

* RGPs before manual inspection

** RGPs after manual inspection (see Materials and Methods)

${ }^{1}$ P. luminescens TT01 and P. asymbiotica ATCC 43949 for Photorhabdus strains; X. bovienii SS-2004 and X. nematophila ATCC19061 for Xenorhabdus strains.

${ }^{2}$ P. luminescens TT01, P. asymbiotica ATCC 43949, X. bovienii SS-2004, X. nematophila ATCC19061

${ }^{3}$ P. luminescens TT01, P. asymbiotica ATCC43949, X. nematophila ATCC19061, X. bovienii SS-2004, Yersinia pestis CO92 (accession number NC_003143); Salmonella enterica subsp. enterica Typhi (accession number NC_003198), Erwinia carotovora SCRI1043 (accession number NC_004547) and E. coli K12 (accession number NC_000913).

genomes of Photorhabdus and Xenorhabdus within Enterobacteriaceae were consistent with the findings of other studies.

The flexible genome was found to be larger in Photorhabdus than in Xenorhabdus genus. This finding is consistent with previous genomic analyses highlighting the importance of genome plasticity in Photorhabdus genomes, at both the species $[36,40,44]$ and clonal [38] levels.

\section{Classification of RGPs}

We classified the RGPs into different classes according to their genetic features (Figure 1.B and Table 2). First, we used the Prophinder tool [53] to identify prophages (P) (Table 2): $\mathrm{Pl}, \mathrm{Pa}, \mathrm{Xn}$ and $\mathrm{Xb}$ have five, eight, six and seven predicted prophages, respectively. P0_PL, P78_PL, P0_PA, P30_PA, P67_XB and P22bis_XN are P2-related phages. This is consistent with previous studies in the Photorhabdus and Xenorhabdus genera in which P2phage tail structures were identified [54-58].

RGPs showing at least one of the typical features of MGEs acquired by HGT (insertion near a tRNA gene, an integrase-coding gene or a $\mathrm{G}+\mathrm{C}$ content different from that of the core genome) and that are not prophages were named GIs. No ICE class was created since no T4SS loci was identified in the four studied genomes. GI85_PL and GI25_PA, in the Pl and Pa genomes, respectively, were found to harbor type three secretion system (T3SS) loci similar to those of Yersinia pestis and Pseudomonas aeruginosa [35,36,40,59]. Many pathogenic Gram-negative bacteria encode T3SSs of the Ysc type [60]. In Pl, this T3SS is involved in bacterial adaptation to the insect host, as it prevents the uptake of bacteria by the immunity organs of Locusta migratoria [61]. As previously described [30,59], no such loci are found in Xenorhabdus genomes. The GI27_PL of Pl is another cluster potentially involved in interactions between bacteria and host, as it harbors a homolog of the Yersinia adhesion pathogenicity island (YAPI) [43]. The YAPI encodes a type IV pilus, which contributes to pathogenicity in Yersinia pseudotuberculosis serotype O:1, but also includes genes encoding proteins involved in general metabolism, a gene cluster for a restrictionmodification system and a large number of mobile genetic elements [62]. This YAPI cluster was detected only on the Pl chromosome. Finally, GI63_XN from Xn is a gene cluster potentially involved in nematode interaction. It harbors the nil locus, enabling $X$. nematophila strains to colonize their nematode host, $S$. carpocapsae, specifically [42]. It also encodes putative peptide

Table 2 Classification of RGPs in the P. luminescens TT01, P. asymbiotica ATCC43949, X. nematophila ATCC19061 and $X$. bovienii SS-2004 genomes as a function of their genetic composition (the proportion of the modules belonging to a given class is indicated in parentheses)

\begin{tabular}{|c|c|c|c|c|}
\hline & \multicolumn{2}{|c|}{ Typical MGE } & \multicolumn{2}{|c|}{ RGP sensu stricto } \\
\hline & Prophages & Genomic islands & $\mathrm{RGP}_{\text {mob }}{ }^{1}$ & $\mathrm{RGP}_{\text {none }}{ }^{2}$ \\
\hline P. luminescens TT01 & $5(5 \%)$ & $38(39,5 \%)$ & $23(24 \%)$ & $30(31 \%)$ \\
\hline P. asymbiotica ATCC 43949 & $8(8,5 \%)$ & $33(36 \%)$ & $10(11 \%)$ & $41(45 \%)$ \\
\hline X. nematophila ATCC19061 & $6(7 \%)$ & $32(38,5 \%)$ & $27(32 \%)$ & $18(22 \%)$ \\
\hline X. bovienii SS-2004 & $7(10 \%)$ & $23(32 \%)$ & $21(30 \%)$ & $20(28 \%)$ \\
\hline
\end{tabular}

${ }^{1}$ RGPs that are not prophages, Gls but encode potential DNA recombination enzymes (resolvase, invertase and excisionase)

${ }^{2} \mathrm{RGPs}$ that are not prophages, Gls or $\mathrm{RGP}_{\text {mob }}$ 
synthetases, which may be involved in antibiotic production, thereby facilitating the eviction of competing bacteria during the association of nematodes and bacteria before the nematodes leave the insect cadaver. The GI63_XN is specific to Xn.

The other predicted regions were named RGPs sensu stricto. Several of these RGPs contained ISs and genes encoding enzymes catalyzing DNA recombination, such as resolvase, invertase and excisionase. We named these RGPs RGP $_{\text {mob }}$ RGPs with no remarkable features were named $\mathrm{RGP}_{\text {none }}$. This last group of RGPs probably consists of hypervariable regions with intracellular mobility mediated by chromosomal rearrangements, such as deletion, duplication or inversion. $\mathrm{RGP}_{\text {mob }}$ and $\mathrm{RGP}_{\text {none }}$ may also be ancient mobilizable MGEs with degraded mobility machinery. The membership to multiple RGP classes of Photorhabdus virulence cassettes (PVCs) and the toxin complex (Tc) loci is consistent with this hypothesis. PVCs are phage-like elements flanked by variable putative or identified toxin genes [63-65]). They are found in Photorhabdus genomes, but not in Xenorhabdus genomes. Yang and coworkers speculated that PVCs might encode phage-like structures, acting as syringes to deliver toxins to the interior of eukaryotic cells [64]. Six of the eight previously described PVC families [36], belong to the prophage and GI classes (PVCcif: GI52_PA, GI60_PL, PVClopT of Pl: GI56_PL, PVCpnf of Pa: GI81_PA, PVClmt of Pa: GI57_PA, PVCphx of Pl and its tandem repeat: $\mathrm{P} 41$ PL). The remaining three families were classified as $\mathrm{RGP}_{\text {mob}}$, despite their putative ancestral origin from phages (PVClopT of $\mathrm{Pa}$ : RGP54_PA, PVCphx of PA: RGP66_PA). The Tc loci of Photorhabdus and Xenorhabdus encoded families of insecticidal toxins active by ingestion $[35,36,39,41]$. These loci are conserved in several other entomopathogenic bacteria (Serratia entomophila, Pseudomonas entomophila) and bacteria associated with insects (Yersinia spp., Pseudomonas syringae) $[43,63,66,67]$, strongly suggesting that HGT of Tc loci has occurred between soil bacteria from different genera known to interact with insects. Some Tc loci are embedded in GIs (GI94_PL, GI27 PL, GI22 PL, GI23 PA, GI91 PA, GI50 XN, GI56_XN, GI23_XB), whereas others are found in $\mathrm{RGP}_{\text {none }}$ (RGP14_PL, RGP58_PL, RGP47_XN, RGP14_XB, RGP30_XB). In these two examples, the genomic erosion of HGT features may lead to a loss of information in some RGPs. This process has classically been reported for nucleotide usage: with increasing time since the HGT event, codon usage in the inserted DNA is gradually modified to match the background of the recipient genome [68]. $R G P_{\text {mob }}$ and $R G P_{\text {none }}$ may also be MGE that can be mobilizable in trans by other MGEs or non canonical MGEs with mobility mechanisms that have yet to be described. Our classification thus opens up promising new lines of research that may lead to the identification of new classes of MGE.

From the viewpoint of whole-genome evolution, our classification highlights the unusual position of the $\mathrm{Pa}$ genome. The proportions of $\mathrm{RGP}_{\text {mob }}$ and $\mathrm{RGP}_{\text {none }}$ are fairly similar in the $\mathrm{Pl}, \mathrm{Xb}$ and $\mathrm{Xn}$ genomes, whereas the $\mathrm{Pa}$ genome contains a much higher proportion of $\mathrm{RGP}_{\text {none }}$ (Table 2). This difference probably results from differences in genome evolution and/or plasticity between $\mathrm{Pa}$, which was recovered from a human patient in North America [32], and the other three strains, which were isolated from nematodes. The evolutionary implications of the higher proportion of $\mathrm{RGP}_{\text {none }}$ in $\mathrm{Pa}$ remain unclear, but a systematic functional analysis of $\mathrm{RGP}_{\text {none }}$ specific to $\mathrm{Pa}$ would complement virulence mapping techniques [29], thereby contributing to the identification of new MGEs involved in the emergence of human pathogens.

\section{RGPs in the core genome architecture}

We mapped the different classes of RGPs on schematic circular maps, to visualize their chromosomal distribution (Additional File 4). GIs, prophages and RGPs sensu stricto were found to be evenly distributed throughout the four genomes. Replication, by its inherent asymmetry, shapes the global structure of the prokaryotic chromosome, and some regions of the chromosome are much less accessible for internal recombination than others [15]. However, GI, prophages and RGPs sensu stricto were equally likely to be located in the region of the origin of replication, the replication termination region or other regions. This permissiveness probably results from compensatory lateral transfers and/or recombination events, preventing dramatic effects on gene order or the large-scale organization of the genome [15].

We searched for RGP location sites by identifying the genes flanking them on the right and left (Additional File 2). We then looked for RGP location sites conserved within the core genome. We identified 108 hotspots conserved in at least three of the studied genomes, including 14 sites located close to a tRNA gene (tRNAsite) and 94 sites located close to a protein-encoding gene (coding sequence or CDS-site). By definition, tRNA attachment sites are associated with GIs and prophages, whereas coding sequence sites may be associated with all classes of RGPs (Table 3). Therefore, CDS-sites are potential recombination hot spots for hypervariable regions or integration hot spots for MGE or ancient mobilizable MGE. CDS-sites have been little described. However, a recent comparative genomic study in Escherichiacoli showed that most gene acquisitions and losses (83\%) are adjacent to CDS-sites rather than tRNA-sites [69]. Similarly to tRNA-sites, primary nucleotide 
Table 3 RGP sites (genes flanking RGPs on the right or left) conserved in the P. luminescens TT01 (PI), P. asymbiotica ATCC43949 (Pa), X. nematophila ATCC19061 (Xn) and X. bovienii strain SS-2004 (Xb) genomes.

\begin{tabular}{|c|c|c|c|c|c|}
\hline & location sites * & $\mathrm{Xn}$ & $\mathrm{Xb}$ & $\mathrm{PI}$ & $\mathrm{Pa}$ \\
\hline & yjeS_tRNA-gly & Gl10 & $\mathrm{Gl7}$ & Gl103 & core genome \\
\hline & ampH_tRNA-leu & Gl89 & Gl10 & Gl100 & P95 \\
\hline & yjdC_tRNA-phe & P75 & Gl23 & Gl94 & $\mathrm{Gl} 23$ \\
\hline & folD_tRNA-arg & P75 & P22bis & Gl95 & $\mathrm{Gl} 25$ \\
\hline & rpoD_tRNA-met & P88 & Gl29 & $\mathrm{Gl} 88$ & P86 \\
\hline & ylaC_tRNA-ans & Gl36 & Gl29 & inside Gl44 & inside GI64 \\
\hline & ghrA_tRNA-ser & Gl45 & Gl33 & $\mathrm{Gl} 49$ & $\mathrm{Gl} 60$ \\
\hline \multirow[t]{20}{*}{ tRNA gene integration site } & pgsA_tRNAleu_tRNAcys & Gl56 & P46 & $\mathrm{Gl} 47$ & Gl61 \\
\hline & yjeM_tRNA-pro & Gl69 & $\mathrm{Gl} 52$ & Gl71 & P39 \\
\hline & mltC_tRNAphe & Gl69 & Gl64 & $\mathrm{Gl} 27$ & $\mathrm{Gl} 81$ \\
\hline & $g l t X \_t R N A-v a l \_2 t R N A l y s \_3 t R N A-v a l$ & Gl71bis & Gl59 & Gl33 & Gl35 \\
\hline & vacJ_tRNA-arg & Gl71bis & inside GI55 & Gl76 & inside Gl35 \\
\hline & rsmC_tRNA-leu & Gl76 & $\mathrm{Gl} 67$ & Gl95 & Gl13 \\
\hline & $y c c K \_t R N A-s e r$ & P43 & Gl16 & $\mathrm{Gl} 44$ & Gl64 \\
\hline & aroH & not present & RGP43 & RGP64 & RGP48 \\
\hline & asmA/hisL & RGP32 & core genome & RGP38 & RGP68 \\
\hline & cheZ & RGP35 & RGP30 & RGP45 & RGP63 \\
\hline & $c p \times A / c y s E$ & $\mathrm{Gl} 2$ & RGP83 & Gl110 & Gl104 \\
\hline & $c p \times P$ & RGP1 & RGP82 & Gl109 & P103 \\
\hline & $\operatorname{csp} E$ & RGP28bis & $\mathrm{Gl} 61$ & RGP31 & RGP62 \\
\hline & deaD & core genome & RGP8 & RGP101 & RGP96 \\
\hline & dnaB/zur & RGP84 & P77 & RGP96 & RGP94 \\
\hline & dnaJ & RGP17 & RGP27 & RGP17 & inside RGP15 \\
\hline & dnaQ & Gl78 & core genome & Gl26 & $\mathrm{Gl} 22$ \\
\hline & $d x s$ & $\mathrm{Gl} 22$ & $\mathrm{Gl} 25$ & core genome & RGP82 \\
\hline & ecfL & P88 & Gl76 & $\mathrm{Gl} 188$ & P86 \\
\hline & eno & $\mathrm{Gl} 20$ & RGP71 & RGP25 & inside GI21 \\
\hline \multirow[t]{23}{*}{ Protein encoding gene integration site } & $e x b D$ & RGP79 & RGP26 & RGP87 & RGP84 \\
\hline & $f b a A$ & RGP65 & RGP58 & $\mathrm{Gl} 27$ & Gl23 \\
\hline & fis & Gl20 & RGP73 & RGP92 & RGP89 \\
\hline & $f l g L$ & GP37 & G 131 & RGP46 & GP62 \\
\hline & $f l g N$ & G 138 & GP30 & GP45 & GP63 \\
\hline & $f l h D$ & G 136 & G 129 & G 144 & Gl64 \\
\hline & fliR & Gl37 & RGP48 & RGP46 & RGP62 \\
\hline & $\operatorname{TfrdA} / y h h Q$ & RGP15 & core genome & RGP93 & RGP90 \\
\hline & $g c v P$ & RGP25 & core genome & $\mathrm{Gl} 181$ & Gl27 \\
\hline & $g \ln G$ & RGP95 & core genome & RGP5 & RGP5 \\
\hline & $g \ln S$ & RGP29 & inside RGP22 & Gl32 & RGP73 \\
\hline & $g \mid p D$ & RGP96bis & RGP2 & Gl3bis & $\mathrm{Gl3}$ \\
\hline & gntX (yhgH) & RGP97 & RGP3 & RGP4 & RGP4 \\
\hline & gyrB/glmS & RGPO & RGPO & Po & PO \\
\hline & hrpA & RGP49bis & RGP38 & RGP52 & $\mathrm{Gl} 57$ \\
\hline & $k d p E$ & RGP29 & RGP22 & Gl33 & RGP73 \\
\hline & leuB & RGP22bis & Gl65 & RGP85bis & core genome \\
\hline & lysS & P26 & Gl64 & P80 & $\mathrm{Gl} 28$ \\
\hline & malM & Gl91 & core genome & Gl12 & Gl10 \\
\hline & $m d t A$ & core genome & RGP48 & Gl68 & RGP43 \\
\hline & mdtC/icd & RGP60 & Gl49 & Gl69 & $\mathrm{Gl} 42$ \\
\hline & $m / t D$ & RGP77 & Gl57 & RGP25 & $\mathrm{Gl} 21$ \\
\hline & $m o b B$ & $\mathrm{Gl91}$ & RGP81 & core genome & RGP8 \\
\hline
\end{tabular}


Table 3: RGP sites (genes flanking RGPs on the right or left) conserved in the P. luminescens TT01 (PI), P. asymbiotica ATCC43949 (Pa), X. nematophila ATCC19061 (Xn) and X. bovienii strain SS-2004 (Xb) genomes. (Continued)

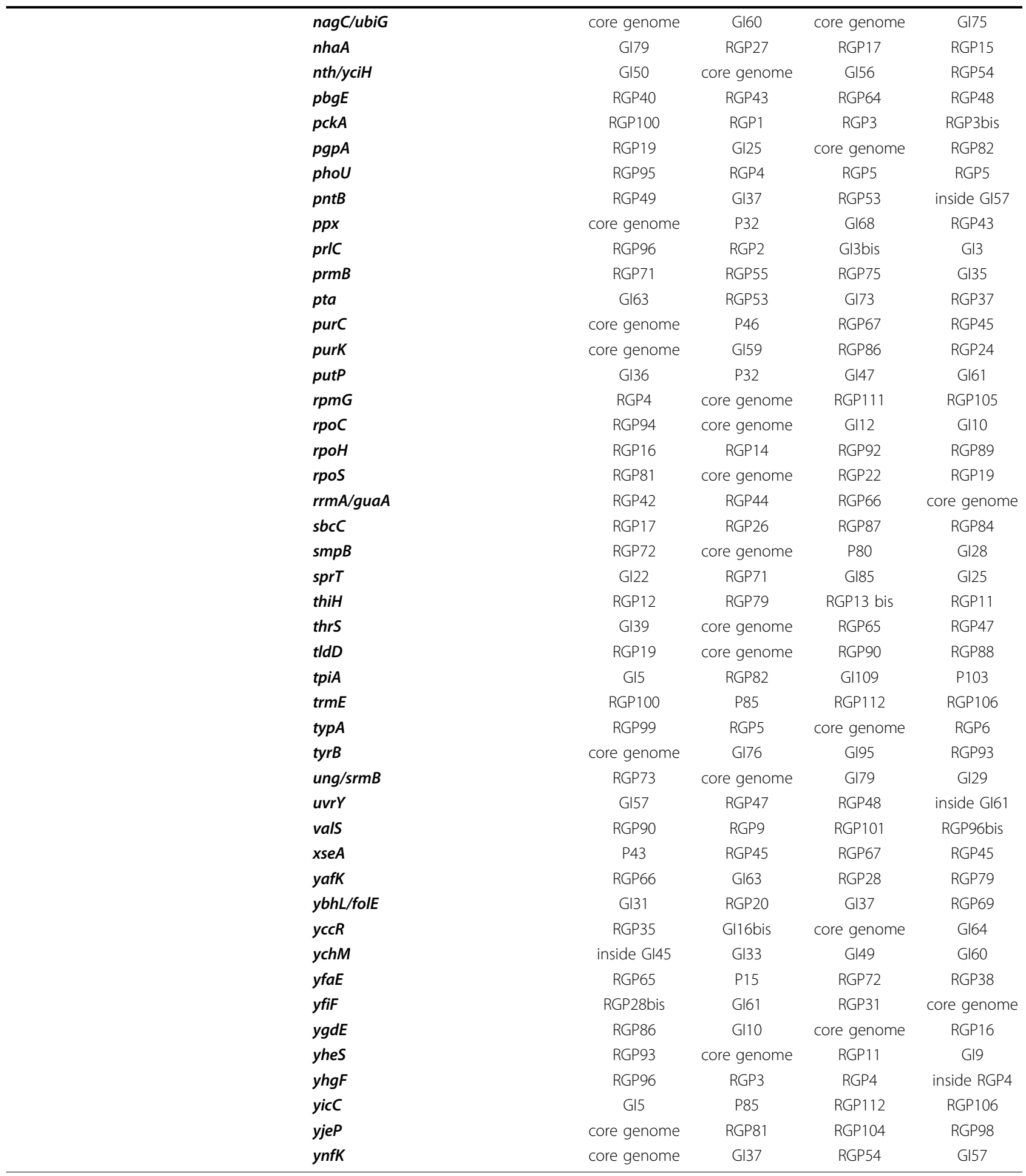

* Iocation sites are listed when they are conserved in at least three of the genomes panel.

When the two RGP flanking genes are conserved, the gene pair is indicated as location site. If note, each flanking gene is considered as potential location site. 
sequences (e.g. repeat sequences) or secondary structures close to the CDS-sites may be targeted as integration hot spots. Alternatively, the function of the neighboring gene may be required for intracellular mobility. Indeed, in our study, the genes encoded at CDS-sites were frequently found to be involved in DNA and RNA metabolism or often encoding transferases (Table 3). They may therefore act as cofactors in the excision/integration process.

Very different RGPs were found to be located in the same integration hot spot, in the different Photorhabdus and Xenorhabdus genomes. For example, we compared the RGPs located within the trmE CDS-site (Figure 2). In the Salmonella enterica Typhimurium genome, trmE is the insertion point for the SG1 genomic island, which includes antibiotic resistance genes and metabolic genes
[70,71]. We found no homolog of SGI1 within the trmE sites of the Photorhabdus and Xenorhabdus genomes or elsewhere in the complete genome sequences for these genera. In Photorhabdus strains, RGP112_PL and RGP106_PA are located in the trmE site. These two RGPs are very similar, differing only in the presence of a large central inversion and additional flanking blocks of genes in Pl. Unlike the Photorhabdus genomes, the Xenorhabdus genomes had different RGPs at the trmE site, RGP100_XN and P85_XB. Some P85_XB gene blocks were found to be located in another region of the $X$. nematophila genome, between the $m r c \mathrm{~A}$ and $c p x \mathrm{P}$ integration sites (RGP1_XN), highlighting the modular structure of the RGP (see below).

In addition to the trmE CDS-site, a number of other integration hotspots are conserved in other bacteria.

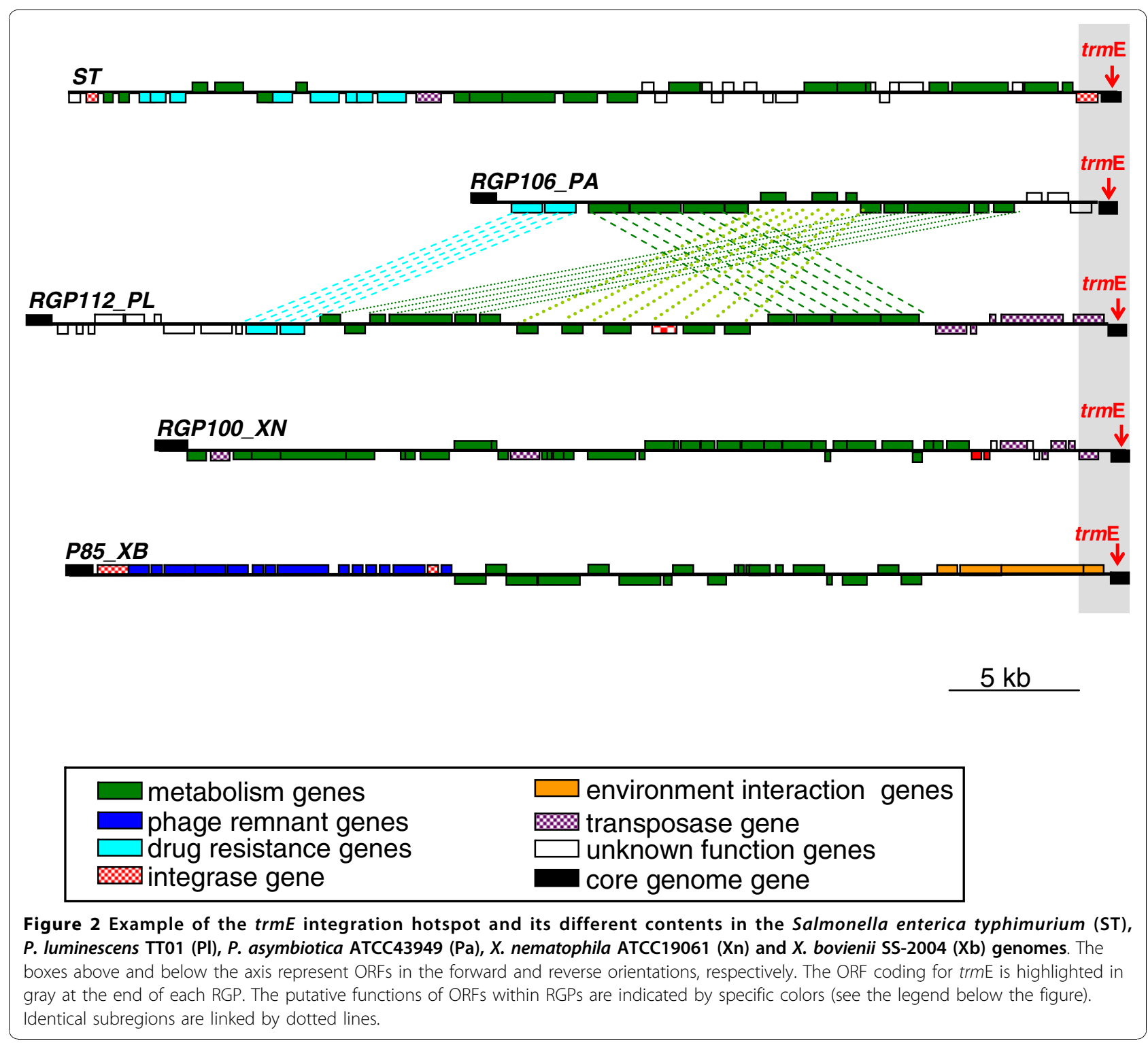


The CS54 genomic island of Salmonella enterica serotype Typhimurium [72] and the $14 \mathrm{~kb}$ genomic island of E. coli CFT073 [73] are both flanked by the $x s e A$ integration hotspot. The rpoS gene is considered to be a recombination hotspot within the Enterobacteriaceae [74]. Integration hotspots are also conserved in more distantly related bacteria. $f l g L$ and $f b a A$ are the integration sites of the flagellin glycosylation island of Pseudomonas aeruginosa [75] and the ICESt1 and ICESt3 of Streptococcus thermophilus [76], respectively. The tropism of MGEs for particular integration hotspots in phylogenetically unrelated taxa highlights the probable ancestral role of such sites in integration and recombination.

\section{Modules are the functional units within RGPs}

We have shown that no single RGP is conserved between the four genomes. Indeed, the RGPs identified were either unique or shared a limited number of subregions. An exhaustive in silico analysis provided evidence for the structuring of each RGP (P, GI, RGP ${ }_{\text {mob }}$ and $\mathrm{RCP}_{\text {none }}$ ) into several subregions or modules. These modules are blocks of genes 0.5 to $60 \mathrm{~kb}$ in length, with a conserved gene order (synteny) in at least two genomes of the Entero set or specific to the strain (see Materials and Methods).

The modular structure of RGPs sensu lato is illustrated by the organization of RGP99_XN in Xn (Figure 3.A). This RGP is located between two genes of the core genome, typ $A$ and treC. RGP99_XN can be broken down into four modules. Two of these modules, RGP99_XN_b and RGP99_XN_c, have a similar organization to gene blocks present in $\mathrm{Xb}, \mathrm{Pa}$ and $\mathrm{Pl}$. The other two modules, RGP99_XN_a and RGP99_XN_d, are specific to the $\mathrm{Xn}$ strain (Figure 3.B). Each module is characterized by a specific $\mathrm{G}+\mathrm{C}$ content. Furthermore, most of the genes within a given module are involved in similar functions: the RGP99_XN_b module encodes a putative type six secretion system (T6SS; [77]), the RGP99_XN_c module encodes the XhlA hemolysin, which has been implicated in virulence in insects [78], and the RGP99_XN_d module encodes proteins that may be involved in DNA recombination.

For each genome, a list of modules is given in Additional File 5 and the module to which each gene belongs is identified in the gene file accessible on PhotoScope and XenorhabduScope (see Materials and Methods). We distinguished eight functional classes of modules: 1) metabolic modules, consisting of genes involved in primary metabolism, metabolite transport and cell component biosynthesis; 2) drug resistance modules; 3) antibiotic synthesis modules encoding bacteriocins, non ribosomal peptide synthetase, polyketide synthases; 4) phage modules, 5) recombination modules encoding enzymes involved in DNA recombination, such as transposase, invertase, excisionase; 6) environment interaction modules encoding proteins or proteinaceous structures involved in interactions with the environment (iron uptake, adhesion to surfaces etc.), 7) host-interaction modules encoding virulence or symbiosis factors essential for interaction with the insect or nematode host; 8) modules of unknown function.

We calculated the proportion of modules belonging to each functional class in the four genomes studied (Figure 4). As previously reported [17], RGPs consist of recombination (4 to $16 \%$ ) and phage (9 to $14 \%$ ) modules, each of which mediate intracellular and intercellular DNA mobilization in Eubacteria. Surprisingly, recombination modules were found to be more frequent in the Xenorhabdus genomes $(13.5 \%$ and $16.5 \%$, in $\mathrm{Xb}$ and $\mathrm{Xn}$, respectively) than in the Photorhabdus genomes ( $4 \%$ and $11 \%$ in $\mathrm{Pa}$ and $\mathrm{Pl}$, respectively). This difference in recombination module content suggests a difference in recombination strategy between the two genera, with greater recombination activity in the Xenorhabdus genomes. This hypothesis is supported by the different patterns of GC skew between the Photorhabdus and Xenorhabdus genomes. Indeed, whereas the Photorhabdus genome GC skews were classical, with two major shifts, one near the origin and the other near the replication termination site, the Xenorhabdus genome GC skews were inverted at several points over the chromosome (Additional File 4). These data were confirmed by optical mapping [34] and pulsed-field gel electrophoresis (Ogier and Gaudriault, unpublished data). Such unusual GC skew patterns have already been described in a few genomes [79-82] and were interpreted as the result of recent chromosomal rearrangements, which are commonly observed in vivo [79].

Antibiotic modules (3 to 6\%) and drug resistance modules ( 4 to $5 \%$ ) constitute another canonical module class $[17,20]$. The Photorhabdus and Xenorhabdus flexible genomes contain numerous genes and operons encoding antibiotic molecules and secondary metabolites, such as non-ribosomal peptides, polyketides and bacterocins. These products are prime candidates for HGT, because they provide a gain-of-function phenotype and are not essential to the microbial cell [2]. Furthermore, one of the major challenges for entomopathogenic nematode symbiosis is the maintenance of a monoxenic infection in an insect cadaver in the soil. Flexibility, resulting in the renewal of antimicrobial factors, is therefore likely to be determinant for Photorhabdus and Xenorhabdus.

In addition to the most common building blocks found in MGEs, we identified modules more specifically involved in metabolism, environment/host interaction and unknown functions. Metabolic modules 


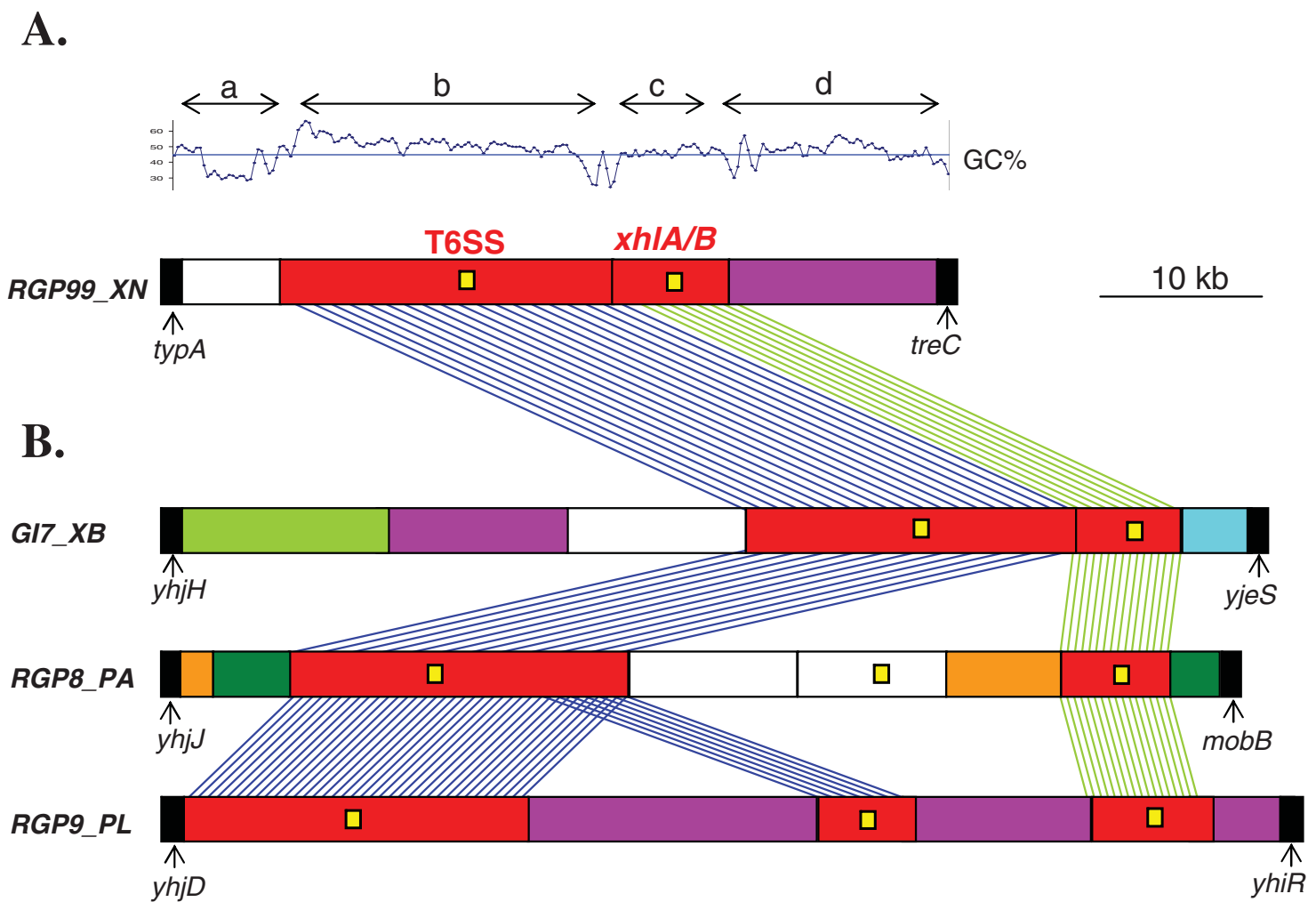

\begin{tabular}{|c|c|c|}
\hline$\square$ metabolism & $\square$ environment interaction & $\begin{array}{l}\square \text { conserverved modules } \\
\text { between genomes }\end{array}$ \\
\hline$\square$ antibiotic synthesis & recombination & \\
\hline$\square$ drug resistance & $\square$ unknown function & \\
\hline host interaction & core genome & \\
\hline
\end{tabular}

Figure 3 Schematic diagram of the modular structure of RGP99_XN in the $X$. nematophila ATCC19061 (Xn) genome and of some RGP99_XN module counterparts in the P. luminescens TT01 (PI), P. asymbiotica ATCC43949 (Pa), X. bovienii SS-2004 (Xb) genomes. A. RGP99_XN can be divided into four subregions or modules, which are distinguished by letters $a, b, c, d$. $G+C$ is indicated above the modules. B. The T6SS (RGP99_XN_b) and XhIA/B (RGP99_XN_c) module counterparts in P. luminescens TT01 (RGP9_PL), P. asymbiotica ATCC43949 (RGP8_PA) and X. bovienii Sj- 2004 (G17_XB). The boxes represent modules, corresponding to blocks of genes specific to the strain or blocks of syntenic genes, (i.e. with a conserved genomic organization in at least two genomes of the Entero set). The putative biological functions of modules are defined by the specific colors of the box (see the legend below the figure). Yellow squares indicate modules conserved in at least two genomes of the Entero set. Others are modules specific to the strain. The thin vertical black arrows indicate RGP integration sites. The conserved modules in the four genomes are linked by dotted lines. Modules are conserved if they have more than $80 \%$ of syntenic genes in common (syntenic genes between two genomes are colocalized genes that shared at least 30\% of identity on $80 \%$ of the shortest sequence by BlastP). For example, the two modules RGP99_XN_b (T6SS) and RGP99_XN_c (Xh/AB) are conserved between the four genomes. RGP99_XN_b is composed of 16 genes potentially encoding a type six secretion system (T6SS). The 16 genes display colocalized orthologous genes in XB, PA and PL (identity between orthologs of XN and XB modules: 75\% to 97\%; identity between orthologs of XN, PL and PA modules: $58 \%$ to $91 \%$ ). RGP99_XN_C is composed of 3 genes, two of them encode an hemolysin belonging to the two partner secretion system family and display colocalized orthologs in XB (73 to $90 \%$ of identity), PA (52 to $75 \%$ of identity) and PL (51 to $77 \%$ of identity).

constituted the most frequently represented class, regardless of the strain considered (22-27\%). This finding highlights the contribution of the acquisition of additional metabolic traits to adaptability and competitiveness under certain circumstances, such as during the colonization of a new niche or rapidly changing growth conditions [2]. Interactions between host and environment also account for a large proportion of modules (22-31\%). This may reflect the complex life style of these bacteria, which interact with two invertebrate hosts, an insect and a nematode. No known function could be attributed to 13 to $18 \%$ of the modules, and the hypothetical proteins encoded by the genes of these modules are good candidates for identifying new genes playing a role in the particular lifestyle of these bacteria. 

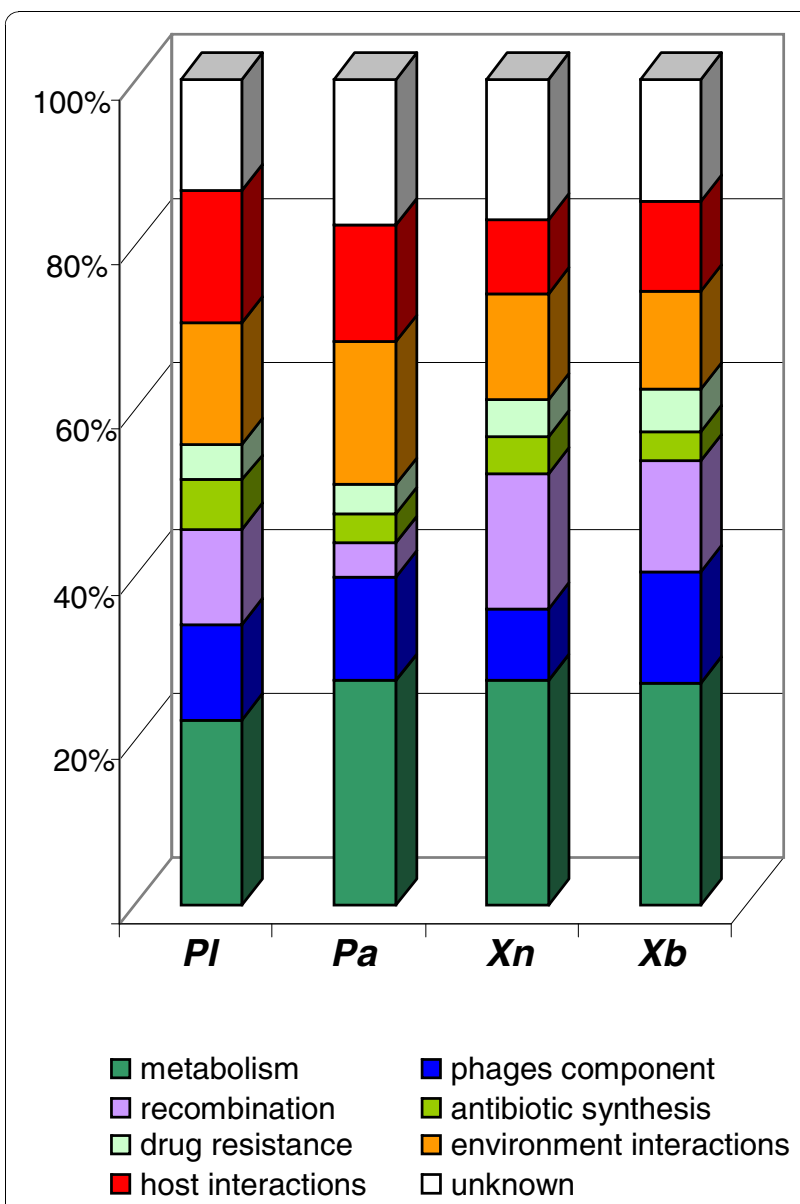

Figure 4 Module function distribution in the flexible genomes of Photorhabdus luminescens TT01 (PI), Photorhabdus asymbiotica ATCC43949 $(\mathrm{Pa})$, Xenorhabdus nematophila ATCC19061 (Xn) and Xenorhabdus bovienii SS-2004 (Xb). Eight module functions were defined (see the legend below the figure).

The modular structure of some MGEs has already been reported in previous studies. This structure consists principally of intracellular mobility modules, intercellular mobility modules and antibiotic resistance modules $[17,20]$. Furthermore, it has been described principally for phages, transposons, ICEs and GIs [24,69,83-89]. By measuring features describing the rate of change of DNA in the locus of enterocyte and effacement of Escherichia coli, Castillo and coworkers also concluded that the GI had a mosaic structure and identified subregions, rather than the whole GI, as the true units of selection [90].

In this study, we show that (i) the modular structure relates not only to prophages and GIs but also to $\mathrm{RGP}_{\text {mob }}$ and $\mathrm{RGP}_{\text {none, }}$ (ii) module functions cover a broad range of functions involved in adaptation to the bacterial environment. RGPs sensu lato are therefore polyfunctional, and the functional unit of the RGPs is the module. This RGP organization is observed in the
Enterobacteriaceae family, but probably also throughout the prokaryotic kingdom.

\section{Modules are the plasticity units of RGPs during long-term genome evolution}

As discussed above, no entire RGP is conserved between the four genomes analyzed in this study and RGPs are composed of functional units, the modules. We investigated whether the mobility of these modules within and between cells could have contributed to shaping the RGPs in the different strains or species of a taxon. We compared the distribution of modules throughout the genome and their organization, between the four genomes studied.

We first analyzed the distribution of modules within the Entero set initially used for RGP characterization (Figure 5). We defined five classes of module as a function of their conservation within a taxonomic group: i) modules found exclusively in the genome of the strain of interest ("strain" modules); ii) modules found exclusively in the genomes of the two Photorhabdus strains or the two Xenorhabdus strains ("genus" modules); iii) modules present in at least one Photorhabdus genome and one Xenorhabdus genome but absent from the genomes of other bacteria of the "Entero" set ("PhotoXeno" modules), iv) modules found in genomes of pathogenic bacteria of the "Entero" set ("pathogens" modules) and v) modules found in the non pathogenic bacterium E. coli K12 strain ("Enterobacteriaceae" modules). The modules belonging to "Photo-Xeno", "Enterobacteriaceae" and "pathogens" groups were evenly distributed in the four strains, together accounting for between 30 and $40 \%$ of the modules (Figure 5). There were relatively few "Photo-Xeno"-specific modules (7\% to $13 \%)$. Therefore, despite having very similar lifestyles (entomopathogenic bacteria living in symbiosis with nematodes) and being closely related phylogenetically, the flexible genomes of Xenorhabdus and Photorhabdus were different, suggesting different mechanisms of molecular adaptation to the environment and hosts of these two genera. Most of the modules in these four genomes were of the "strain" and "genus" types, these two types of module together accounting for about two thirds of the modules. However, the Photorhabdus genus had similar proportions of "strain" and "genus" modules, whereas the Xenorhabdus genus had a higher proportion of "strain" modules (54 to 57\%) than of "genus" modules ( $9 \%$ to $11 \%$ ). Thus, the flexible genome of Xenorhabdus, unlike that of Photorhabdus, is mostly strain-specific. This may reflect the closer phylogenetic relationship between $P$. luminescens and $P$. asymbiotica than between $X$. nematophila and X. bovienii [26].

We then assessed module synteny between the four strains studied, by aligning conserved modules on linear 


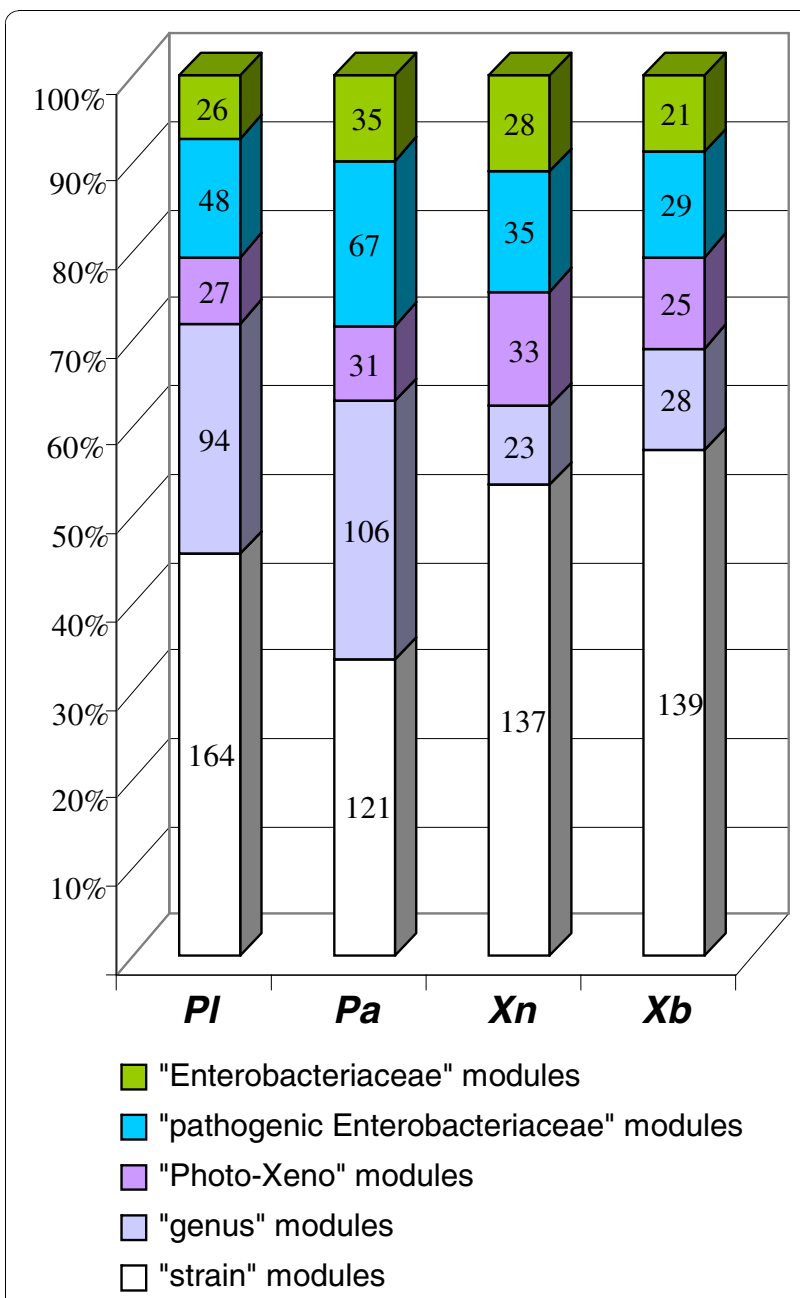

Figure 5 Distribution of the $P$. luminescens TT01 (PI), $P$. asymbiotica ATCC43949 (Pa), $X$. nematophila ATCC19061 (Xn) and $X$. bovienii SS-2004 (Xb) modules among five subsets: i) modules found exclusively in the genome of the strain of interest ("strain" modules); ii) modules found exclusively in the genomes of the two Photorhabdus strains or the two Xenorhabdus strains

("genus" modules); iii) modules present in at least one Photorhabdus genome and one Xenorhabdus genome but absent from genomes of other bacteria of the "Entero" set ("Photo-Xeno" modules), iv) modules found in the genomes of pathogenic bacteria of the "Entero" set ("pathogenic Enterobacteriacae" modules) and v) modules found in the non pathogenic E. coli K12 strain ("Enterobacteriaceae" modules).

genomic maps (Figure 6). Studied strains share little module synteny with each other, particularly Xenorhab$d u s$ species. As an illustration of this absence of synteny between modules, we further analyzed the genomic organization of modules initially described in RGP99_XN, in the four genomes studied (Figure 3). In the $\mathrm{Xn}, \mathrm{Pl}, \mathrm{Pa}$ and $\mathrm{Xb}$ genomes, the T6SS and XhlA/B modules were both found in a single RGP but they are not located in the same integration site, as these modules were flanked by different genes of the core genome.
The T6SS and XhlA/B modules are either in a syntenic block $(\mathrm{Xn}$ and $\mathrm{Xb})$, intercalated with additional modules ( $\mathrm{Pa}$ and $\mathrm{Pl})$ or partially duplicated $(\mathrm{Pl})$. They are also flanked by additional modules specific to the strain or conserved inside others RGPs. A patchy structure has already been described for MGEs, consistent with horizontal transfer leading to the gradual stepwise construction of these MGEs [69,90-92].

Our data show that the RGPs of the flexible genome are shaped by the acquisition and loss of modules, and that RGP diversity probably results from intrachromosomal or interchromosomal rearrangements between module units.

\section{Modules are the units of deletion of RGPs involved in short-term genome rearrangements}

If modules do indeed shape the RGPs during genome evolution, some rearrangements are likely to be detectable in clonal populations during growth in the laboratory. We therefore searched for intrachromosomal rearrangements of modules within the genome of Pl clonal variants available in our laboratory. TT01 $\alpha$ is a Pl variant collected from a laboratory-maintained symbiotic nematode [38]. TT01 $\alpha$ differs from the Pl reference genome by nine large-scale deletion events in the flexible genome, but these genomic changes have cryptic phenotypic consequences. The boundaries of the deleted regions in TT01 $\alpha$ were located in the Pl genome by a combination of macrorestriction and DNA microarray experiments [38].

We first compared the boundaries of the deleted regions and the module boundaries. All the regions deleted in TT01 $\alpha$ were found to be embedded in an RGP, and seven of the nine regions matched with one or several modules. We analyzed the precise boundaries of four selected loci within these seven deleted regions: locus D, which matches module RGP45_PL_a and encodes metabolism and drug resistance functions; locus E, which matches module RGP53_PL_c and encodes antibiotic biosynthesis functions; locus $\mathrm{F}$, which matches with modules GI59_PL_a, b and encodes metabolism and host interaction functions; locus I, which matches with modules P80_PL a, b, c, d, e, f and encodes metabolism and phage functions [38]. For each locus, we performed multiplex PCR amplification with two primer pairs flanking the left (primer 1/primer 2) and the right (primer 3/primer 4) boundaries of the regions deleted in TT01 $\alpha$ (Figure 7.A). As predicted by macrorestriction and DNA microarray experiments, PCR amplification generated two fragments (fragments [1-2] and [3-4]) when Pl genomic DNA was used as the template, and one fragment (fragment [1-4]) when TT01 $\alpha$ genomic DNA was used as the template (Figure 7.B), confirming

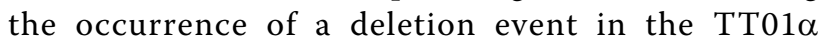




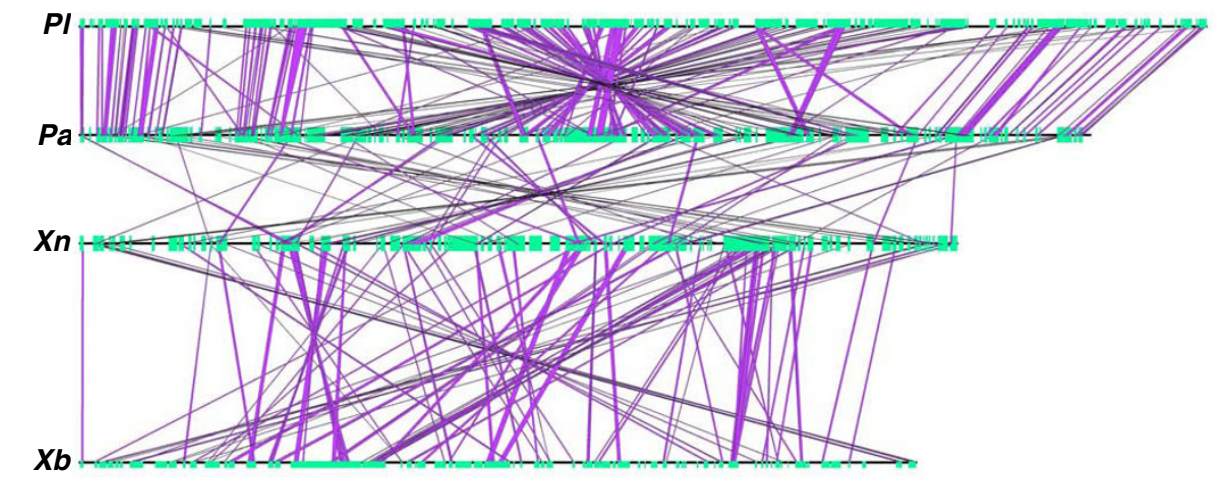

Figure 6 Schematic representation of the genomic organization (synteny) of "genus" and "Photo-Xeno" modules conserved between P. luminescens TT01 (PI), P. asymbiotica ATCC43949 (Pa), X. nematophila ATCC19061 (Xn) and X. bovienii SS-2004 (Xb). Each genome is represented by a horizontal line, with the RGPs sensu lato represented as green boxes. Purple lines indicate the conservation of modules between two adjacent chromosomes(the representation is dependent on chromosome order on the figure, i.e. a module conserved between $X$. bovienii and P. luminescens will not be drawn).

genome. We mapped the deletion boundaries more precisely, with the goal of obtaining an exact picture of the result of the deletion making it possible to predict the intrachromosomal rearrangements occurring at these loci, by sequencing the four fragments [1-4] obtained from the TT01 $\alpha$ genome.

For all four fragments, the sequence data confirmed the predicted deletion boundaries: the D, E, F, I locus boundaries exactly matched the module boundaries identified by in silico analysis, validating our modularization procedure. Moreover, we distinguished two classes of deletion patterns potentially matching at least three deletion scenarios (Figure 8). At loci $\mathrm{F}$ and I, which are embedded in GIs, the whole module or block of modules was found to be missing in the TT01 $\alpha$ genome, suggesting that a single block deletion event led to the Pl/TT01 $\alpha$ transition in this part of the genome. In both cases, a gene encoding an enzyme involved in DNA recombination (transposase for locus $\mathrm{F}$ and integrase for locus I) is located at the internal border of the locus and is therefore a good candidate for involvement in the rearrangement. By contrast, at loci D and E, which are embedded in RGPs sensu stricto, only subregions of modules are missing in the TT01 $\alpha$ genome, consistent with the occurrence of more complex rearrangement events in these genomic areas during the $\mathrm{Pl} / \mathrm{TT} 01 \alpha$ transition. Locus $\mathrm{E}$ in the TT01 $\alpha$ genome consists of five fragmented remnants of the initial locus E. Genomic reduction has probably occurred in several stages at locus E, although the absence of mobile elements or genes encoding recombination functions makes it difficult to determine the genomic rearrangement scenario. Locus $\mathrm{D}$ in the TT01 $\alpha$ genome displays a shuffling pattern at three locations: a 105 bp nucleotide sequence at the left internal border; a region composed of two Enterobacteriaceae repeat intergenic consensus (ERIC) sequences, a transposase-encoding gene and a nucleotidic sequence of $154 \mathrm{bp}$ at the right internal border; a region composed of the truncated plu1870 and plu1872 genes and the plu1871 gene interrupted by a transposase gene in the middle part. As the elements within these shuffled subregions are probably remnants of molecular actors involved in the rearrangements of locus D, we analyzed them further. ERIC elements are miniature (127 bp) non autonomous mobile elements in Enterobacteriaceae genomes [93,94]. The Pl genome is particularly rich in such repeats [35]. The two transposase genes encoded a transposase of the IS 928 family. The 105 and 154 bp sequences are palindromic nucleotide sequences consisting of fragments dispersed in the $\mathrm{Pl}$ genome. They display no sequence similarity to each other or to ERIC and the two transposase genes. The inserted transposases and ERIC sequences clearly played a role in the plasticity of locus D, but the origin and role of the exogenous $105 \mathrm{bp}$ and 154 bp nucleotide sequences remain unknown.

In conclusion, whatever the molecular mechanism involved in these deletion scenarios, in the case of clonal genomic plasticity, the modules may be deleted over a timescale corresponding to growth in the laboratory and may be considered units of deletion within RGPs. 


\section{A.}
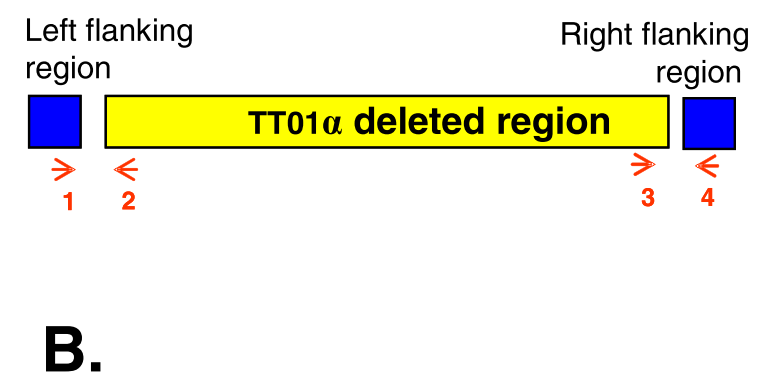

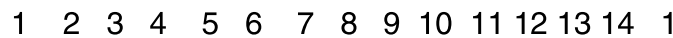

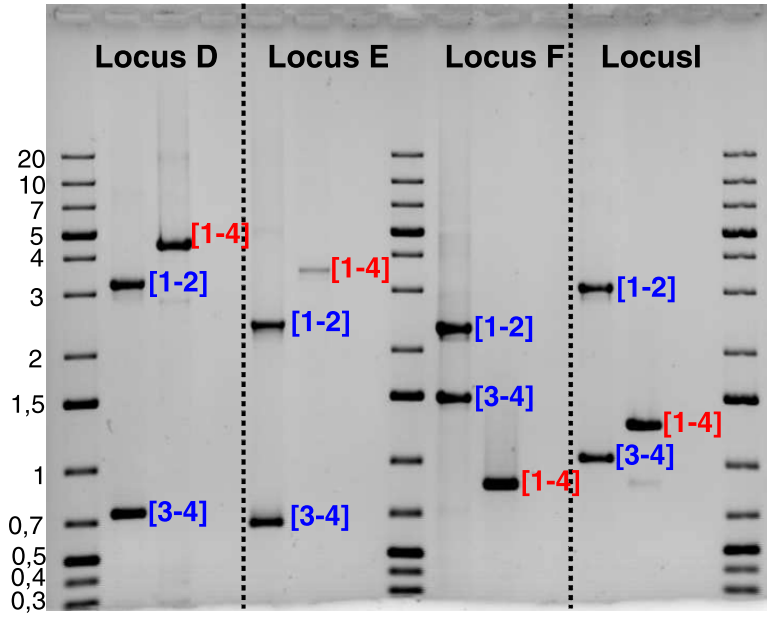

Figure 7 Boundaries of deleted regions in the genome of the $P$. luminescens $T T 01 \alpha$ variant match with the module boundaries defined in the $P$. luminescens TT01 (PI) genome. A. Schematic diagram of the strategy used for multiplex PCR amplification. The yellow box indicates the deleted region. Blue boxes represent the flanking regions. Red horizontal arrows indicate the location of the primers. The primer mixture (P1, P2, P3 and P4) was designed to amplify two fragments from the PI genome and only one fragment if the region is effectively deleted, as predicted for the P. luminescens TT01 $\alpha$ variant genome. B. Agarose gel electrophoresis of the PCR products generated by amplifying the genomic DNA of PI (lanes 2, 5, 9, 12), of the TT01 $\alpha$ variant (lanes 3 , $6,10,13$ ) or of water (lanes $4,7,11,14$ ) with primers P1, P2, P3 and $\mathrm{P} 4$, designed as indicated in (A) for loci D (lanes 2-4), E (lanes 5-7), F (lanes 9-11) and I (lanes 12-14). Loci D, E, F and I are four regions of the $\mathrm{Pl}$ genome identified as missing in the $\Pi \pi 01 \alpha$ variant genome (see text for details). [1-2], [3-4] and [1-4]. indicate bands of sizes compatible with amplification of the regions between the P1 and $\mathrm{P} 2$ primers, the $\mathrm{P} 3$ and $\mathrm{P} 4$ primers and the $\mathrm{P} 1$ and $\mathrm{P} 4$ primers, respectively. Lanes 1, 8, 15: molecular markers. The sizes of fragments are indicated in $\mathrm{kb}$ to the left of the gel.

\section{Conclusions}

The data presented here participate to a better vision of the bacterial flexible genome organization. The characterization of RGPs by the RGPFinder method showed the flexible genome to be much broader than the sum of GIs and prophage elements. Additional elements $\mathrm{RGP}_{\text {mob }}$ and $\mathrm{RGP}_{\text {none }}$ elements - lacking classical mobility features may be hypervariable regions that undergo deletions, ancient mobile elements with a degraded mobilization machinery, MGE that can be mobilizable in trans by other MGEs or non canonical MGE for which the mobility mechanism has yet to be described. Furthermore, we provide evidence that not only GIs and prophages, but all RGPs sensu lato have a mosaic structure composed of modules that are both functional and plasticity units.

The application of comparative genome sequencing to experimental evolution studies provides us with an opportunity to study the link between genome dynamics and adaptive evolution. Nevertheless, such studies are generally carried out on bacterial populations evolving in a synthetic broth culture, and they mostly identify point mutations $[95,96]$. Here, by carrying out comparative genomics studies on variants obtained from their host in the laboratory, we showed experimentally that the same modules undergo genomic rearrangements during genome speciation and short-term genomic rearrangements. This work improves our understanding of the process responsible for bacterial genome diversification and evolution.

Obtaining of these data were made possible by the use of the Photorhabdus and Xenorhabdus genera for our comparative genomic study. Indeed, the life cycle of these genera is restricted to two successive ecological niches. We argue that this unusual pattern of selective pressure is responsible for an alternation of genomic shuffling: HGT in the insect cadaver, which constitutes an abundant nutrient resource potentially shared with many other microorganisms and intrachromosomal rearrangements of recently acquired modules in the bacterial monoxenic culture within the nematode gut, as observed in the Pl variant isolated from a laboratorymaintained symbiotic nematode. We therefore suggest that Photorhabdus and Xenorhabdus are suitable new bacterial models for studies of the evolution of bacterial genomes.

Finally, the data obtained in this study contribute to our understanding of the fluid nature of genomes throughout the kingdoms of life. According to J. A. Shapiro, prokaryotic and eukaryotic cells are genetic engineers and mobile elements are "natural genetic engineering systems" that facilitate the evolutionary rewriting of genomic information [97]. Shapiro's hypothesis is that repeated evolutionary challenges have selected systems that (i) reduce the size of the genomic search space and (ii) maximize the chance of success by using combinatorial processes based on basal functional components [97]. We argue that the modules described here are entirely consistent with this vision, as these 


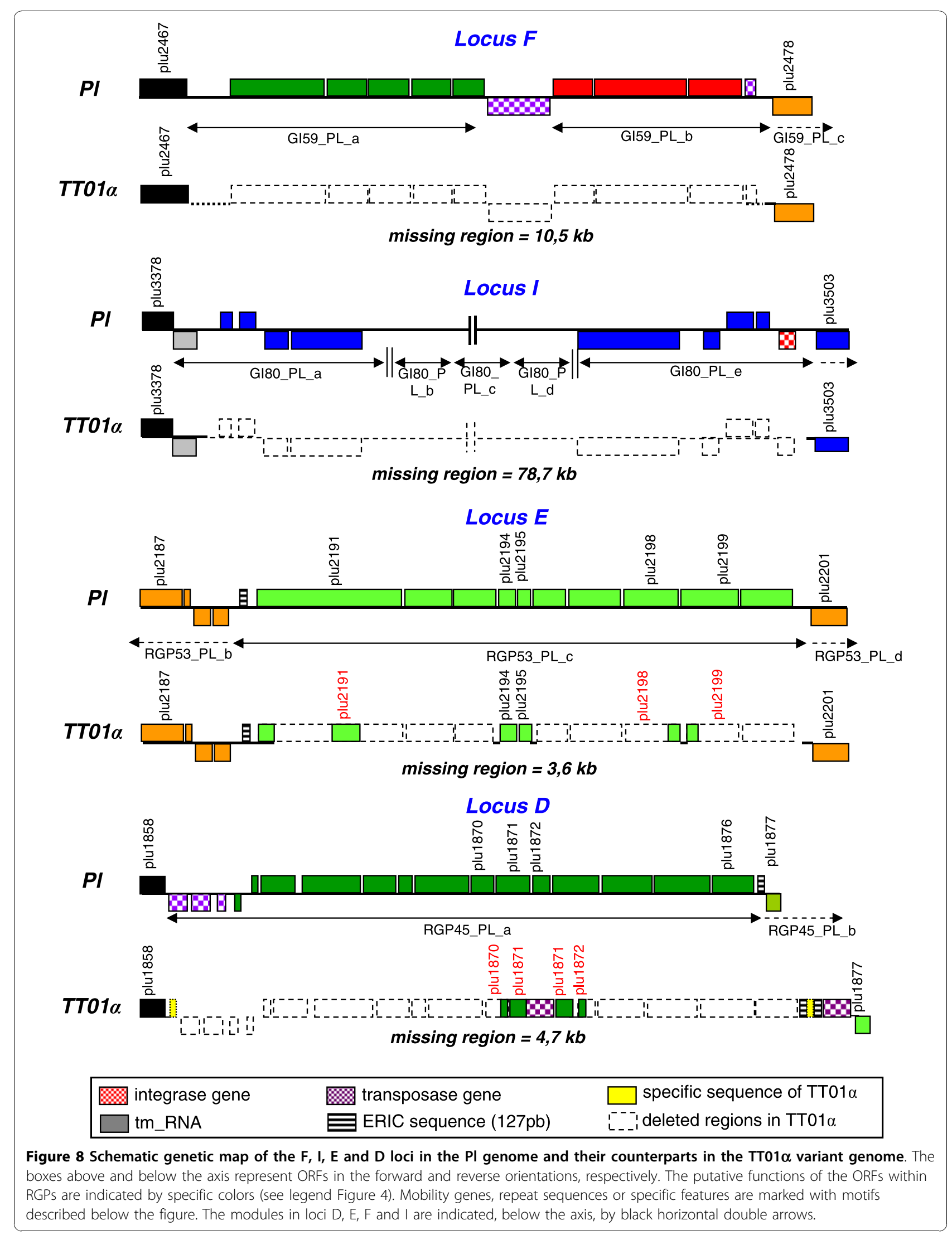


functional units recombined at a limited number of hotspots shaping and delimiting the flexible genome.

\section{Methods}

Bacterial strains and genome sequences

Photorhabdus luminescens subspecies laumondii strain TT01 is a symbiont of the nematode Heterorhabditis bacteriophora isolated in Trinidad and Tobago [35,98]. The genome of strain TT01 consists of a single circular chromosome of 5,688,987 bp (accession number NC_005126). Photorhabdus asymbiotica subspecies asymbiotica strain ATCC43949 is a North American clinical isolate. This strain was isolated in 1977 from a female patient with endocarditis, in Maryland, USA $[32,99]$. The genome of strain ATCC43949 consists of a single circular chromosome of $5,064,808 \mathrm{bp}$ and a 29,732 bp plasmid [36] (accession numbers NC_012962 and NC_012961, respectively). Xenorhabdus nematophila ATCC19061, the type strain of the species, is a symbiont of the nematode Steinernema carpocapsae, isolated from Georgia, USA [100]. The genome of strain ATCC19061 comprises a single circular chromosome of 4,432,590 bp and a 155,327 bp plasmid [34] (accession numbers FN667742 and FN667743, respectively). Xenorhabdus bovienii SS-2004 is a symbiont of the nematode Steinernema jollieti sp. isolated from a woodland in the Missouri valley, USA, in 1999 [101]. The genome of strain SS-2004 comprises a single circular chromosome of 4,225,498 bp [34] (accession number FN667741). The four genomes were input into the PhotoScope and XenorhabduScope databases http://www.genoscope.cns. fr/agc/mage.

\section{RGP identification}

Regions of genomic plasticity (RGPs) were sought in the P. luminescens TT01, P. asymbiotica ATCC43949, X. nematophila ATCC19061 and X. bovienii SS-2004 genomes, with the RGPfinder web tool implemented in the MaGe annotation platform (http://www.genoscope.cns. $\mathrm{fr} / \mathrm{agc} / \mathrm{mage}$; Roche et al., unpublished data). Briefly, RGPFinder searches for breaks in synteny between a reference genome and the genomes of a set of related bacteria - the bacterial genome set (Figure 1.A). A RGP sensu lato is the sum of overlapping subregions missing in at least one of the bacterial genomes of the comparison set. RGPs have a minimal size of $5 \mathrm{~kb}$. This excludes the isolated insertion sequences of the RGPs, but favors regions with several genes of potential functional interest in the bacterial biology. This definition does not involve any underlying assumption about the evolutionary origin or genetic basis of these variable chromosomal segments. RGPFinder also provides information about composition abnormalities (GC\% deviation, codon adaptation index) and about the features flanking the RGPs, such as tRNA, IS, integrase (int) and genetic elements involved in DNA mobility (mob), which are common characteristics of foreign DNA acquired by horizontal genetic transfer. The results obtained with this web tool include those for Alien Hunter [102], a method detecting atypical sequences $(i$. $e$., sequences potentially acquired by horizontal genetic transfer) through the analysis of composition bias.

Predicted RGPs were then manually inspected, to eliminate false-positive results. Indeed, point mutations may lead RGPFinder to identify a region as an RGP when it actually belongs to the core genome. Finally, the boundaries of the RGP were homogenized between the compared genomes and potential insertion sites were defined. The genomes used in the bacterial genome set were those of $P$. luminescens TT01, $P$. asymbiotica ATCC43949, X. nematophila ATCC19061, X. bovienii SS-2004, Yersinia pestis CO92 (accession number 003143); Salmonella enterica subsp. enterica Typhi CT18 (accession number NC_003198), Erwinia carotovora subsp. atroseptica SCRI1043 (accession number NC_004547) and E. coli K12 (accession number NC_000913). Finally, Prophinder was used to detect prophages among the RGP sensu lato [53], http://aclame. ulb.ac.be/Tools/Prophinder/.

\section{Definition and distribution of modules}

The MaGe web interface [103] was used to divide RGPs manually into subregions corresponding to blocks of genes specific to the strain or blocks of syntenic genes (i.e. genes with a conserved genomic organization in at least two genomes of the Entero set). These subregions, which often contain genes of similar biological function, were named "modules". The distribution of modules among the "Enterobacteriaceae" genome set was analyzed manually: a module was considered present (or partially present) in a genome if it had more than $80 \%$ (25\%) of syntenic orthologous genes (orthologous genes shared at least $30 \%$ of identity on $80 \%$ of the shortest sequence by BlastP) with the module of the reference genome. The module was otherwise considered to be absent. Descriptions of the modules and their distributions are available from PhotoScope https://www.genoscope.cns.fr/agc/mage/wwwpkgdb/Login/log.php?pid=13 and XenorhabduScope https://www.genoscope.cns.fr/ agc/mage/wwwpkgdb/Login/log.php?pid=24, by opening the Genomic Object Editor of a gene and consulting the "Module" results.

\section{Multiplex PCR procedure and sequencing}

Genomic DNA was extracted as previously described [44] and stored at $4^{\circ} \mathrm{C}$. Primers flanking the right (primers P1/P2) and left (primers P3/P4) module borders were designed with Primer 3 http://frodo.wi.mit.edu/ 
primer3/. Primer sequences are listed in Additional File 6. Multiplex PCR with the four primers (P1, P2, P3, P4) was performed with a Bio-Rad thermocycler (Bio-Rad, Marne La Vallée, France). Fragments with a predicted size smaller than $3 \mathrm{~kb}$ were amplified with Invitrogen Taq polymerase (Invitrogen, France), according to the manufacturer's protocol. Fragments with a predicted size greater than $3 \mathrm{~kb}$ were amplified with the Herculase Enhanced DNA polymerase (Stratagene, Amsterdam Zuidoost, Pays Bas) in accordance with the manufacturer's recommendations. Samples of reaction mixtures were analyzed by electrophoresis in an agarose gel. The fragments amplified by PCR [P3-P4] were purified from the gel with the high purity purification kit from Roche (Roche Diagnostic, France) and sequenced with the PCR primers described in Additional File 6, via a chromosome walking process, by Macrogen (South Korea).

\section{Additional material}

\begin{abstract}
Additional File 1: Phylogenetic tree for the Enterobacteriaceae derived from a distance analysis of 16S rRNA gene sequences. The genomes used in this study belong to species indicated in red (Photorhabdus and Xenorhabdus) and blue (other Enterobacteriaceae). Vibrio cholerae (Vibrionaceae) was used as an outgroup. The GenBank accession numbers of the sequences are shown in brackets. Bootstrap values of more than $50 \%$ are indicated at the nodes. The bar indicates $1 \%$ sequence divergence. A figure showing a phylogenetic tree for the Enterobacteriaceae used in this study.
\end{abstract}

Additional File 2: List of regions of genomic plasticity (RGPs) in the $P$. luminescens TT01 (PI), P. asymbiotica ATCC43949 (Pa), $X$. nematophila ATCC19061 (Xn) and X. bovienii SS- $2004(\mathrm{Xb})$ genomes. A table listing the RGPs.

Additional File 3: Distribution of RGP sizes in the Photorhabdus luminescens TT01 (PI), Photorhabdus asymbiotica ATCC43949 (Pa), Xenorhabdus nematophila ATCC19061 (Xn) and Xenorhabdus bovienii SS-2004 genomes (Xb). A figure showing the distribution of RGP size.

Additional File 4: Schematic diagram of the distribution of RGPs sensu lato on the circular chromosomes of $P$. luminescens TT01 (PI), $P$. asymbiotica ATCC43949 (Pa), X. nematophila ATCC19061 (Xn) and $X$. bovienii SS-2004 (Xb). Successive circles from inside to outside: GC skew; GC deviation (with values exceeding +/- 2 standard deviations indicated in red). Distribution of the different RGP types: Gls (orange), Phages (green) and RGP mob and RGP none (yellow). A figure showing schematic diagrams of the distribution of RGPS.

Additional File 5: List of modules in the $P$. luminescens TT01 (PI), $P$. asymbiotica ATCC43949 (Pa), X. nematophila ATCC19061 (Xn) and X. bovienii SS-2004 (Xb) genomes and their distribution in the Yersinia pestis CO92 (Yp), Salmonella enterica subsp. enterica Typhi CT18 (St), Erwinia carotovora subsp. atroseptica SCRI1043 (Eca) and E. coli K12 (Eco) genomes. A table listing the modules.

Additional File 6: Primers used in the study. A table listing the primers used in this study.

\section{List of abbreviations}

Eco: Escherichia coli K12; Eca: Erwinia carotovora subsp. atroseptica SCRI1043; Gl: genomic island; HGT: horizontal genetic transfer; ICE: integrative conjugative element; int: integrase; IS: insertion sequence; MGE: mobile genetic element; P: prophage; Pa: Photorhabdus asymbiotica ATCC43949; PI:
Photorhabdus luminescens TT01; PVC: Photorhabdus virulence cassette; RGP region of genomic plasticity; St : Salmonella enterica subsp. enterica Typhi CT18; T3SS : type three secretion system; T6SS: type six secretion system; Tc: toxin complex; Xn: Xenorhabdus nematophila ATCC19061; Xb: Xenorhabdus bovienii SS-2004; YAPI: Yersinia adhesion pathogenicity island; Yp: Yersinia pestis $\mathrm{CO} 2$

\section{Acknowledgements}

We thank Christine Laroui for technical assistance. We thank the Xenorhabdus genome consortium for access to the Xenorhabdus genomes, R. Ffrench-constant and N. Waterfield for access to the Photorhabdus asymbiotica genomes before public access. We thank Eric Duchaud for critical reading of parts of the manuscript. This study received financial support from the Institut National de la Recherche Agronomique (grant SPE 2007_1133_03), the Agence Nationale de la Recherche (ANR PFTV MicroScope) and the GIS IBiSA.

\section{Author details}

${ }^{1}$ INRA, UMR 1133, Laboratoire EMIP, Place Eugène Bataillon, F-34095 Montpellier, France. ${ }^{2}$ Université Montpellier 2, UMR 1133, Laboratoire EMIP, Place Eugène Bataillon, F-34095 Montpellier, France. ${ }^{3}$ CEA, Genoscope \& CNRS-UMR 8030, Laboratoire d'Analyse Bioinformatique en Génomique et Métabolisme, Evry cedex F-91006, France. ${ }^{4}$ Department of Biological Sciences, University of Wisconsin, Milwaukee, WI 53201, USA. ${ }^{5}$ Department of Bacteriology, University of Wisconsin, Madison, WI 53706 USA. ${ }^{6}$ Great Lakes Bioenergy Research Center, University of Wisconsin, Madison, WI 53706 USA.

\section{Authors' contributions}

J-CO, AG, CM, PT, SG designed the project. SF, HG-B, GS provided genomic data. JCO carried out in silico description of the RGPS, their modularization and the PCR experiments. AC, DR, ZR contributed in computational analysis. J-CO, AC, RZ, AG, PT, CM, SG analyzed the data. J-CO and SG wrote the paper with contributions from AG. All authors read and approved the final manuscript.

Received: 19 March 2010 Accepted: 15 October 2010 Published: 15 October 2010

\section{References}

1. Hacker J, Carniel E: Ecological fitness, genomic islands and bacterial pathogenicity - A Darwinian view of the evolution of microbes. EMBO Reports 2001, 2(5):376-381.

2. Dobrindt $U$, Hochhut $B$, Hentschel $U$, Hacker J: Genomic islands in pathogenic and environmental microorganisms. Nature Reviews Microbiology 2004, 2(5):414-424.

3. Abby S, Daubin V: Comparative genomics and the evolution of prokaryotes. Trends in Microbiology 2007, 15(3):135-141.

4. Frost LS, Leplae R, Summers AO, Toussaint A: Mobile genetic elements: The agents of open source evolution. Nature Reviews Microbiology 2005, 3(9):722-732.

5. Mathee K, Narasimhan G, Valdes C, Qiu X, Matewish JM, Koehrsen M, Rokas A, Yandava CN, Engels R, Zeng E, et al: Dynamics of Pseudomonas aeruginosa genome evolution. Proceedings of the National Academy of Sciences of the United States of America 2008, 105(8):3100-3105.

6. Rocha EPC: Order and disorder in bacterial genomes. Current Opinion in Microbiology 2004, 7(5):519-527.

7. Burrus V, Pavlovic G, Decaris B, Guedon G: Conjugative transposons: the tip of the iceberg. Molecular Microbiology 2002, 46(3):601-610.

8. Roberts AP, Chandler M, Courvalin P, Guédon G, Mullany P, Pembroke T, Rood JI, Smith CJ, Summers AO, Tsuda M, et al: Revised nomenclature for transposable genetic elements. Plasmid 2008, 60(3):167-173.

9. Beaber JW, Hochhut B, Waldor MK: SOS response promotes horizontal dissemination of antibiotic resistance genes. Nature 2004 427(6969):72-74.

10. Qiu XY, Gurkar AU, Lory S: Interstrain transfer of the large pathogenicity island (PAPI-1) of Pseudomonas aeruginosa. Proceedings of the National Academy of Sciences of the United States of America 2006, 103(52):19830-19835

11. Ramsay JP, Sullivan JT, Stuart GS, Lamont IL, Ronson CW: Excision and transfer of the Mesorhizobium loti R7A symbiosis island requires an 
integrase IntS, a novel recombination directionality factor RdfS, and a putative relaxase RIxS. Molecular Microbiology 2006, 62(3):723-734.

12. Maiques E, Ubeda C, Tormo MA, Ferrer MD, Lasa I, Novick RP, Penades JR: Role of staphylococcal phage and SaPl integrase in intra- and interspecies SaPI transfer. Journal of Bacteriology 2007, 189(15):5608-5616.

13. Juhas M, Crook DW, Dimopoulou ID, Lunter G, Harding RM, Ferguson DJ, Hood DW: Novel type IV secretion system involved in propagation of genomic islands. Journal of Bacteriology 2007, 189(3):761-771.

14. Ravatn R, Zehnder AJB, van der Meer JR: Low-frequency horizontal transfer of an element containing the chlorocatechol degradation genes from Pseudomonas sp. strain B13 to Pseudomonas putida F1 and to indigenous bacteria in laboratoryscale activated-sludge microcosms. Applied and Environmental Microbiology 1998, 64(6):2126-2132.

15. Rocha EPC: The Organization of the Bacterial Genome. Annual Review of Genetics 2008, 42:211-233.

16. Canchaya C, Fournous $G$, Brussow H: The impact of prophages on bacterial chromosomes. Molecular Microbiology 2004, 53(1):9-18.

17. Toussaint A, Merlin C: Mobile elements as a combination of functional modules. Plasmid 2002, 47(1):26-35.

18. Hendrix RW: Bacteriophage genomics. Current Opinion in Microbiology 2003, 6(5):506-511

19. Engelberg-Kulka H, Glaser G: Addiction modules and programmed cell death and antideath in bacterial cultures. Annual Review of Microbiology 1999, 53:43-70.

20. Mazel D: Integrons: agents of bacterial evolution. Nature Reviews Microbiology 2006, 4(8):608-620.

21. Boucher Y, Labbate M, Koenig JE, Stokes HW: Integrons: mobilizable platforms that promote genetic diversity in bacteria. Trends in Microbiology 2007, 15(7):301-309.

22. Pavlovic G, Burrus V, Gintz B, Decaris B, Guedon G: Evolution of genomic islands by deletion and tandem accretion by site-specific recombination: ICESt1-related elements from Streptococcus thermophilus. Microbiology 2004, 150:759-774

23. Burrus V, Waldor MK: Shaping bacterial genomes with integrative and conjugative elements. Research in Microbiology 2004, 155(5):376-386.

24. Brochet M, Rusniok C, Couve E, Dramsi S, Poyarts C, Trieu-Cuot P, Kunst F, Glaser P: Shaping a bacterial genome by large chromosomal replacements, the evolutionary history of Streptococcus agalactiae. Proceedings of the National Academy of Sciences of the United States of America 2008, 105(41):15961-15966.

25. Garriss G, Waldor MK, Burrus V: Mobile Antibiotic Resistance Encoding Elements Promote Their Own Diversity. Plos Genetics 2009, 5(12): e1000775

26. Tailliez P, Laroui C, Ginibre N, Paule A, Pages S, Boemare N: Phylogeny of Photorhabdus and Xenorhabdus based on universally conserved proteincoding sequences and implications for the taxonomy of these two genera. Proposal of new taxa: $X$. vietnamensis sp. nov., P. luminescens subsp. caribbeanensis subsp. nov., $P$. luminescens subsp. hainanensis subsp. nov., P. temperata subsp. khanii subsp. nov., P. temperata subsp. tasmaniensis subsp. nov., and the reclassification of $P$. luminescens subsp. thracensis as $P$. temperata subsp. thracensis. International journal of systematic and evolutionary microbiology 2009.

27. Boemare $\mathrm{N}$ : Interactions between the partners of the entomopathogenic bacterium nematode complexes, Steinernema-Xenorhabdus and Heterorhabditis- Photorhabdus. Nematology 2002, 4:601-603.

28. Forst S, Dowds B, Boemare N, Stackebrandt E: Xenorhabdus and Photorhabdus spp.: Bugs that kill bugs. Annual Review of Microbiology 1997, 51:47-72.

29. Waterfield NR, Sanchez-Contreras M, Eleftherianos I, Dowling A, Wilkinson P, Parkhill J, Thomson N, Reynolds SE, Bode HB, Dorus S, et al: Rapid Virulence Annotation (RVA): Identification of virulence factors using a bacterial genome library and multiple invertebrate hosts. Proceedings of the National Academy of Sciences of the United States of America 2008, 105(41):15967-15972.

30. Goodrich-Blair H, Clarke DJ: Mutualism and pathogenesis in Xenorhabdus and Photorhabdus: two roads to the same destination. Molecular Microbiology 2007, 64(2):260-268.

31. Richards GR, Goodrich-Blair H: Masters of conquest and pillage: Xenorhabdus nematophila global regulators control transitions from virulence to nutrient acquisition. Cellular Microbiology 2009, 11(7):1025-1033
32. Farmer $J J$, Jorgensen $J H$, Grimont PAD, Akhurst RJ, Poinar GO, Ageron E, Pierce GV, Smith JA, Carter GP, Wilson KL, et al: Xenorhabdus luminescens (DNA Hybridization Group-5) from Human Clinical Specimens. Journal of Clinical Microbiology 1989, 27(7):1594-1600.

33. Gerrard J, Waterfield N, Vohra R, ffrench-Constant R: Human infection with Photorhabdus asymbiotica: an emerging bacterial pathogen. Microbes and Infection 2004, 6(2):229-237.

34. Latreille P, Norton S, Goldman BS, Henkhaus J, Miller N, Barbazuk B, Bode HB, Darby C, Du ZJ, Forst $S$, et al: Optical mapping as a routine tool for bacterial genome sequence finishing. BMC Genomics 2007, 8:321.

35. Duchaud E, Rusniok C, Frangeul L, Buchrieser C, Givaudan A, Taourit S, Bocs S, Boursaux-Eude C, Chandler M, Charles JF, et al: The genome sequence of the entomopathogenic bacterium Photorhabdus luminescens. Nature Biotechnology 2003, 21(11):1307-1313.

36. Wilkinson P, Waterfield NR, Crossman L, Corton C, Sanchez-Contreras M, Vlisidou I, Barron A, Bignell A, Clark L, Ormond D, et al: Comparative genomics of the emerging human pathogen Photorhabdus asymbiotica with the insect pathogen Photorhabdus luminescens. BMC Genomics 2009, 10:302.

37. Moran NA, Plague GR: Genornic changes following host restriction in bacteria. Current Opinion in Genetics \& Development 2004, 14(6):627-633.

38. Gaudriault S, Pages S, Lanois A, Laroui C, Teyssier C, Jumas-Bilak E, Givaudan A: Plastic architecture of bacterial genome revealed by comparative genomics of Photorhabdus variants. Genome Biology 2008, 9(7):R117.

39. Waterfield NR, Bowen DJ, Fetherston JD, Perry RD, ffrench-Constant RH: The tc genes of Photorhabdus: a growing family. Trends in Microbiology 2001, 9(4):185-191.

40. Waterfield NR, Daborn PJ, french-Constant RH: Genomic islands in Photorhabdus. Trends in Microbiology 2002, 10(12):541-545.

41. Sergeant M, Baxter L, Jarrett P, Shaw E, Ousley M, Winstanley C, Morgan JAW: Identification, typing, and insecticidal activity of Xenorhabdus isolates from entomopathogenic nematodes in United Kingdom soil and characterization of the xpt toxin loci. Applied and Environmental Microbiology 2006, 72(9):5895-5907.

42. Cowles CE, Goodrich-Blair H: The Xenorhabdus nematophila nilABC genes confer the ability of Xenorhabdus spp. to colonize Steinernema carpocapsae nematodes. Journal of Bacteriology 2008, 190(12):4121-4128.

43. Heermann R, Fuchs TM: Comparative analysis of the Photorhabdus luminescens and the Yersinia enterocolitica genomes: uncovering candidate genes involved in insect pathogenicity. BMC Genomics 2008, 9:40.

44. Gaudriault S, Duchaud E, Lanois A, Canoy AS, Bourot S, DeRose R, Kunst F, Boemare N, Givaudan A: Whole-genome comparison between Photorhabdus strains to identify genomic regions involved in the specificity of nematode interaction. Journal of Bacteriology 2006, 188(2):809-814.

45. Langille MGI, Hsiao WWL, Brinkman FSL: Detecting genomic islands using bioinformatics approaches. Nature Reviews Microbiology 2010, 8(5):372-382.

46. Thomson NR, Clayton DJ, Windhorst D, Vernikos G, Davidson S, Churcher C, Quail MA, Stevens M, Jones MA, Watson M, et al: Comparative genome analysis of Salmonella Enteritidis PT4 and Salmonella Gallinarum 287/91 provides insights into evolutionary and host adaptation pathways. Genome Research 2008, 18(10):1624-1637.

47. Cazalet C, Rusniok C, Bruggemann H, Zidane N, Magnier A, Ma L, Tichit M, Jarraud S, Bouchier C, Vandenesch F, et al: Evidence in the Legionella pneumophila genome for exploitation of host cell functions and high genome plasticity. Nature Genetics 2004, 36(11):1165-1173.

48. Silby MW, Cerdeno-Tarraga AM, Vernikos GS, Giddens SR, Jackson RW, Preston GM, Zhang XX, Moon CD, Gehrig SM, Godfrey SAC, et al: Genomic and genetic analyses of diversity and plant interactions of Pseudomonas fluorescens. Genome Biology 2009, 10(5):R51.

49. Amadou C, Pascal G, Mangenot S, Glew M, Bontemps C, Capela D, Carrere S, Cruveiller S, Dossat C, Lajus A, et al: Genome sequence of the beta-rhizobium Cupriavidus taiwanensis and comparative genomics of rhizobia. Genome Research 2008, 18(9):1472-1483.

50. Lima WC, Van Sluys MA, Menck CFM: Non-gamma-proteobacteria gene islands contribute to the Xanthomonas genome. Omics-a Journal of Integrative Biology 2005, 9(2):160-172. 
51. Lefebure T, Stanhope MJ: Evolution of the core and pan-genome of Strepococcus: positive selection, recombination, and genome composition. Genome Biology 2007, 8(5):R71

52. Gu JY, Wang YF, Lilburn T: A Comparative Genomics, Network-Based Approach to Understanding Virulence in Vibrio cholerae. Journal of Bacteriology 2009, 191(20):6262-6272.

53. Lima-Mendez G, Van Helden J, Toussaint A, Leplae R: Prophinder: a computational tool for prophage prediction in prokaryotic genomes. Bioinformatics 2008, 24(6):863-865.

54. Boemare NE, Boyergiglio MH, Thaler JO, Akhurst RJ, Brehelin M: Lysogeny and Bacteriocinogeny in Xenorhabdus nematophilus and Other Xenorhabdus spp. Applied and Environmental Microbiology 1992, 58(9):3032-3037.

55. Baghdiguian $\mathrm{S}$, Boyergiglio $\mathrm{MH}$, Thaler JO, Bonnot $\mathrm{G}$, Boemare $\mathrm{N}$ : Bacteriocinogenesis in Cells of Xenorhabdus nematophilus and Photorhabdus luminescens - Enterobacteriaceae Associated with Entomopathogenic Nematodes. Biology of the Cell 1993, 79(2):177-185.

56. Thaler JO, Baghdiguian S, Boemare N: Purification and Characterization of Xenorhabdicin, a Phage Tail-Like Bacteriocin, from the Lysogenic Strain F1 of Xenorhabdus nematophilus. Applied and Environmental Microbiology 1995, 61(5):2049-2052.

57. Bondi M, Messi P, Sabia C, Contri MB, Manicardi G: Antimicrobial properties and morphological characteristics of two Photorhabdus luminescens strains. Microbiologica 1999, 22(2):117-127.

58. Gaudriault S, Thaler JO, Duchaud E, Kunst F, Boemare N, Givaudan A: Identification of a P2-related prophage remnant locus of Photorhabdus luminescens encoding an R-type phage tail-like particle. FEMS Microbiology Letters 2004, 233(2):223-231.

59. Brugirard-Ricaud K, Givaudan A, Parkhill J, Boemare N, Kunst F, Zumbihl R, Duchaud E: Variation in the effectors of the type III secretion system among Photorhabdus species as revealed by genomic analysis. Journal of Bacteriology 2004, 186(13):4376-4381.

60. Cornelis GR: The type III secretion injectisome. Nature Reviews Microbiology 2006, 4(11):811-825.

61. Brugirard-Ricaud K, Duchaud E, Givaudan A, Girard PA, Kunst F, Boemare N, Brehelin M, Zumbihl R: Site-specific antiphagocytic function of the Photorhabdus luminescens type III secretion system during insect colonization. Cellular Microbiology 2005, 7(3):363-371.

62. Collyn F, Billault A, Mullet C, Simonet M, Marceau M: YAPI, a new Yersinia pseudotuberculosis pathogenicity island. Infection and Immunity 2004, 72(8):4784-4790

63. Hurst MR, Glare TR, Jackson TA, Ronson CW: Plasmid-located pathogenicity determinants of Serratia entomophila, the causal agent of amber disease of grass grub, show similarity to the insecticidal toxins of Photorhabdus luminescens. Journal of Bacteriology 2000, 182(18):5127-5138.

64. Yang G, Dowling AJ, Gerike U, ffrench-Constant RH, Waterfield NR: Photorhabdus virulence cassettes confer injectable insecticidal activity against the wax moth. Journal of Bacteriology 2006, 188(6):2254-2261.

65. Jubelin G, Chavez CV, Taieb F, Banfield MJ, Samba-Louaka A, Nobe R, Nougayrede JP, Zumbihl R, Givaudan A, Escoubas JM, et al: Cycle inhibiting factors (CIFs) are a growing family of functional cyclomodulins present in invertebrate and mammal bacterial pathogens. PLoS One 2009, 4(3): e4855.

66. Buell CR, Joardar V, Lindeberg M, Selengut J, Paulsen IT, Gwinn ML, Dodson RJ, Deboy RT, Durkin AS, Kolonay JF, et al: The complete genome sequence of the Arabidopsis and tomato pathogen Pseudomonas syringae pv. tomato DC3000. Proceedings of the National Academy of Sciences of the United States of America 2003, 100(18):10181-10186.

67. Vodovar N, Vallenet D, Cruveiller S, Rouy Z, Barbe V, Acosta C, Cattolico L, Jubin C, Lajus A, Segurens B, et al: Complete genome sequence of the entomopathogenic and metabolically versatile soil bacterium Pseudomonas entomophila. Nature Biotechnology 2006, 24(6):673-679.

68. Mann S, Chen YP: Bacterial genomic G + C composition-eliciting environmental adaptation. Genomics 2010, 95(1):7-15.

69. Touchon M, Hoede C, Tenaillon O, Barbe V, Baeriswyl S, Bidet P, Bingen E, Bonacorsi S, Bouchier C, Bouvet O, et al: Organised genome dynamics in the Escherichia coli species results in highly diverse adaptive paths. PLOS Genetics 2009, 5(1):e1000344.

70. Doublet B, Golding GR, Mulvey MR, Cloeckaert A: Potential integration sites of the Salmonella genomic island 1 in Proteus mirabilis and other bacteria. Journal of Antimicrobial Chemotherapy 2007, 59(4):801-803.
71. Doublet B, Golding G, Mulvey M, Cloeckaert A, Fairhead C: Secondary Chromosomal Attachment Site and Tandem Integration of the Mobilizable Salmonella Genomic Island 1. PLOS ONE 2008, 3(4):e2060.

72. Kingsley RA, Humphries AD, Weening EH, Zoete MRd, Winter $S$, Papaconstantinopoulou A, Dougan G, umler AJB: Molecular and Phenotypic Analysis of the CS54 Island of Salmonella enterica Serotype Typhimurium: Identification of Intestinal Colonization and Persistence Determinants. Infection and Immunity 2003, 71(2):629-640.

73. Schouler C, Koffmann F, Amory C, Leroy-Sétrin S, Moulin-Schouleur M: Genomic subtraction for the identification of putative new virulence factors of an avian pathogenic Escherichia coli strain of $\mathrm{O} 2$ serogroup. Microbiology 2004, 150(Pt 9):2973-2984.

74. Martinez-Garcia E, Tormo A, Navarro-Lloréns JM: Polymorphism in the yclCrpoS region of enterobacteria. Current Microbiology 2003, 46(5):365-370.

75. Arora SK, Bangera M, Lory S, Ramphal R: A genomic island in Pseudomonas aeruginosa carries the determinants of flagellin glycosylation. Proceedings of the National Academy of Sciences of the United States of America 2001, 98(16):9342-9347.

76. Bellanger $X$, Roberts AP, Morel C, Choulet F, Pavlovic G, Mullany P, Decaris $B$, Guédon $G$ : Conjugative transfer of the integrative conjugative elements ICESt1 and ICESt3 from Streptococcus thermophilus. Journal of Bacteriology 2009, 191(8):2764-2775.

77. Shrivastava S, Mande SS: Identification and Functional Characterization of Gene Components of Type VI Secretion System in Bacterial Genomes. Plos One 2008, 3(8):e2955.

78. Cowles KN, Goodrich-Blair H: Expression and activity of a Xenorhabdus nematophila haemolysin required for full virulence towards Manduca sexta insects. Cellular Microbiology 2005, 7(2):209-219.

79. Parkhill J, Wren BW, Thomson NR, Titball RW, Holden MTG, Prentice MB, Sebaihia M, James KD, Churcher C, Mungall KL, et al: Genome sequence of Yersinia pestis, the causative agent of plague. Nature 2001, 413(6855):523-527.

80. Wu M, Sun LV, Vamathevan J, Riegler M, Deboy R, Brownlie JC, McGraw EA, Martin W, Esser C, Ahmadinejad N, et al: Phylogenomics of the reproductive parasite Wolbachia pipientis wMel: a streamlined genome overrun by mobile genetic elements. PLoS Biology 2004, 2(3):E69.

81. Klasson L, Andersson SGE: Strong asymmetric mutation bias in endosymbiont genomes coincide with loss of genes for replication restart pathways. Molecular Biology and Evolution 2006, 23(5):1031-1039.

82. Bentley SD, Corton C, Brown SE, Barron A, Clark L, Doggett J, Harris B, Ormond D, Quail MA, May G, et al: Genome of the actinomycete plant pathogen Clavibacter michiganensis subsp sepedonicus suggests recent niche adaptation. Journal of Bacteriology 2008, 190(6):2150-2160.

83. Lescat M, Calteau A, Hoede C, Barbe V, Touchon M, Rocha E, Tenaillon O, Medigue C, Johnson JR, Denamur E: A Module Located at a Chromosomal Integration Hot Spot Is Responsible for the Multidrug Resistance of a Reference Strain from Escherichia coli Clonal Group A. Antimicrobial Agents and Chemotherapy 2009, 53(6):2283-2288.

84. Lloyd AL, Henderson TA, Vigil PD, Mobley HL: Genomic Islands of Uropathogenic Escherichia coli Contribute to Virulence. Journal of Bacteriology 2009, 191(11):3469-3481.

85. Tumapa S, Holden MTG, Vesaratchavest M, Wuthiekanun V, Limmathurotsakul D, Chierakul W, Feil EJ, Currie BJ, Day NPJ, Nierman WC, et al: Burkholderia pseudomallei genome plasticity associated with genomic island variation. BMC Genomics 2008, 9:190.

86. He X, Ou HY, Yu Q, Zhou X, Wu J, Liang J, Zhang W, Rajakumar K, Deng Z: Analysis of a genomic island housing genes for DNA S-modification system in Streptomyces lividans 66 and its counterparts in other distantly related bacteria. Molecular Microbiology 2007, 65(4):1034-1048.

87. Konczy P, Ziebell K, Mascarenhas M, Choi A, Michaud C, Kropinski AM, Whittam TS, Wickham M, Finlay B, Karmali MA: Genomic O island 122, locus for enterocyte effacement, and the evolution of virulent verocytotoxin-producing Escherichia coli. Journal of Bacteriology 2008, 190(17):5832-5840

88. Chouikha I, Germon P, Bree A, Gilot P, Moulin-Schouleur M, Schouler C: A selC-associated genomic island of the extraintestinal avian pathogenic Escherichia coli strain BEN2908 is involved in carbohydrate uptake and virulence. Journal of Bacteriology 2006, 188(3):977-987.

89. Hacker J, Kaper JB: Pathogenicity islands and the evolution of microbes. Annual Review of Microbiology 2000, 54:641-679. 
90. Castillo A, Eguiarte LE, Souza V: A genomic population genetics analysis of the pathogenic enterocyte effacement island in Escherichia coli: The search for the unit of selection. Proceedings of the National Academy of Sciences of the United States of America 2005, 102(5):1542-1547.

91. Sandner L, Eguiarte LE, Navarro A, Cravioto A, Souza V: The elements of the focus of enterocyte effacement in human and wild mammal isolates of Escherichia coli: evolution by assemblage or disruption? Microbiology 2001, 147:3149-3158.

92. Van Houdt R, Monchy S, Leys N, Mergeay M: New mobile genetic elements in Cupriavidus metallidurans $\mathrm{CH} 34$, their possible roles and occurrence in other bacteria. Antonie Van Leeuwenhoek International Journal of General and Molecular Microbiology 2009, 96(2):205-226.

93. Delihas N: Small mobile sequences in bacteria display diverse structure/ function motifs. Molecular Microbiology 2008, 67(3):475-481

94. De Gregorio E, Silvestro G, Petrillo M, Carlomagno MS, Di Nocera PP: Enterobacterial repetitive intergenic consensus sequence repeats in yersiniae: genomic organization and functional properties. Journal of Bacteriology 2005, 187(23):7945-7954.

95. Herring CD, Raghunathan A, Honisch C, Patel T, Applebee MK, Joyce AR, Albert TJ, Blattner FR, van den Boom D, Cantor CR, et al: Comparative genome sequencing of Escherichia coli allows observation of bacterial evolution on a laboratory timescale. Nature Genetics 2006, 38(12):1406-1412.

96. Barrick JE, Yu DS, Yoon SH, Jeong H, Oh TK, Schneider D, Lenski RE, Kim JF: Genome evolution and adaptation in a long-term experiment with Escherichia coli. Nature 2009, 461(7268):1243-U1274.

97. Shapiro JA: Letting Escherichia coli Teach Me About Genome Engineering. Genetics 2009, 183(4):1205-1214.

98. Fischer-Le Saux M, Viallard V, Brunel B, Normand P, Boemare NE: Polyphasic classification of the genus Photorhabdus and proposal of new taxa: $P$ luminescens subsp luminescens subsp nov., P-luminescens subsp akhurstii subsp nov., P-luminescens subsp laumondii subsp nov., P.temperata sp nov., P-temperata subsp temperata subsp nov and P-asymbiotica sp nov. International Journal of Systematic Bacteriology 1999, 49:1645-1656.

99. Akhurst RJ, Boemare NE, Janssen PH, Peel MM, Alfredson DA, Beard CE: Taxonomy of Australian clinical isolates of the genus Photorhabdus and proposal of Photorhabdus asymbiotica subsp asymbiotica subsp nov and P-asymbiotica subsp australis subsp nov. International Journal of Systematic and Evolutionary Microbiology 2004, 54:1301-1310.

100. Poinar GO Jr, Thomas GM: A new bacterium, Achromobacter nematophilus sp. nov. (Achromobacteriaceae: Eubacteriales), associated with a nematode. International Bulletin of Bacteriological Nomenclature and Taxonomy 1965, 15:249-252.

101. Spiridonov SE, Krasomil-Osterfeld K, Moens M: Steinernema jollieti sp $n$. (Rhabditida: Steinernematidae), a new entomopathogenic nematode from the American midwest. Russian Journal of Nematology 2004, 12(1):85-95.

102. Vernikos GS, Parkhill J: Interpolated variable order motifs for identification of horizontally acquired DNA: revisiting the Salmonella pathogenicity islands. Bioinformatics 2006, 22(18):2196-2203.

103. Vallenet D, Labarre L, Rouy Z, Barbe V, Bocs S, Cruveiller S, Lajus A, Pascal G, Scarpelli C, Medigue C: MaGe: a microbial genome annotation system supported by synteny results. Nucleic Acids Research 2006, 34(1):53-65.

doi:10.1186/1471-2164-11-568

Cite this article as: Ogier et al:: Units of plasticity in bacterial genomes: new insight from the comparative genomics of two bacteria interacting with invertebrates, Photorhabdus and Xenorhabdus. BMC Genomics 2010 11:568.

\section{Submit your next manuscript to BioMed Central and take full advantage of:}

- Convenient online submission

- Thorough peer review

- No space constraints or color figure charges

- Immediate publication on acceptance

- Inclusion in PubMed, CAS, Scopus and Google Scholar

- Research which is freely available for redistribution

Submit your manuscript at www.biomedcentral.com/submit 